\title{
Compensation method for complex hysteresis characteristics on piezoelectric actuator based on Separated Level-loop Prandt|-Ishlinskii model
}

\section{Yixiao Yang}

Shenyang Jianzhu University

Dong An

Shenyang Jianzhu University

\section{Ying Xu}

Guangdong University of Technology

Meng Shao

Shenyang Jianzhu University

Yupeng Li ( $\nabla$ lyp@sjzu.edu.cn )

Shenyang Jianzhu University

\section{Research Article}

Keywords: Piezoelectric ceramics actuator, Inverse piezoelectric effect, Nonlinear characteristics, Algorithm identification, Feedforward control compensation

Posted Date: February 24th, 2021

DOl: https://doi.org/10.21203/rs.3.rs-194482/v1

License: (c) (1) This work is licensed under a Creative Commons Attribution 4.0 International License. Read Full License 


\title{
Compensation method for complex hysteresis characteristics on piezoelectric actuator based on Separated Level-loop Prandtl-Ishlinskii model
}

\author{
Yixiao Yang ${ }^{1} \cdot$ Dong $\mathrm{An}^{1} \cdot$ Ying $\mathrm{Xu}^{2} \cdot$ Meng Shao $^{1} \cdot$ Yupeng $\mathrm{Li}^{1 *}$ \\ ${ }^{1}$ School of Mechanical Engineering, Shenyang Jianzhu University, Shenyang 110168, \\ China \\ ${ }^{2}$ School of Electro-mechanical Engineering, Guangdong University of Technology, \\ Guangdong 510006, China \\ *lyp@sjzu.edu.cn
}

Piezoelectric ceramic actuators exhibit nonlinear hysteresis characteristics owing to their material properties. To modify the inverse piezoelectric effect as an ideal linear execution, the classical PrandtlIshlinskii (PI) model is usually used for compensation by feedforward control. The PI model compensates well on simple hysteresis characteristics. However, when the output requirements are complex, the PI model demonstrates uneven compensation accuracy on the complex hysteresis characteristics and cannot achieve an accuracy similar to that of simple hysteresis. This paper proposes a simplification of complex hysteresis: Separated Level-loop PI (SLPI) model. First, we use a loopseparation logic algorithm to simplify the complex hysteresis characteristics to obtain hysteresis in the form of single loops with loop levels and vertexes. Second, the hysteresis characteristics of each loop are independently modeled using the PI model. Finally, the inverse model is reconstructed using a rollback method to restore a positive sequence of the feedforward voltage; then, the feedforward voltage is input as a compensation value to achieve higher and more uniform accuracy. Experiments and discussions show that the SLPI model can effectively improve the compensation results of complex hysteresis characteristics; moreover, the average compensation accuracy difference between single hysteresis loops was reduced.

Piezoelectric ceramics actuator Inverse piezoelectric effect $\cdot$ Nonlinear characteristics $\cdot$ Algorithm identification. Feedforward control compensation 


\section{Introduction}

Owing to the inverse piezoelectric effect of piezoelectric materials, piezoelectric ceramic actuators demonstrate the advantages of nanometer precision and fast response $[1,2]$. The effect is widely used in precision measurement, micro/nanoprocessing, nanopositioning, and other precision mechanical engineering fields [3-6]. However, the nonlinear output exhibited by the inherent characteristics of piezoelectric materials affects the accuracy of the piezoelectric actuator [7]. The hysteresis characteristic is the most important factor that leads to nonlinear characteristics [8].

To correct the nonlinear characteristics to an ideal linear state, several international scholars have conducted extensive research on the feedforward hysteresis compensation in piezoelectric actuators [9]. Establishing differential equations and operator models are common phenomenological modeling solutions [10-13]. The classical Prandtl-Ishlinskii (PI) model is a type of operator model, which has the advantages of a simple structure and only few parameters in the inversion process; moreover, it has a wide range of applications $[14,15]$. The PI model is formed by the weighted superposition of the Play operators. Although the Play operator can describe the characteristics of the hysteresis well, its symmetry limits the modeling accuracy of the PI model [16]. The improved classical PI models apply methods such as generalized models and the parameters increase in the models to maintain the hysteresis while eliminating the symmetry; consequently, the compensation accuracy can be improved to a certain extent [17]. In previous studies, a segmented PI model was also used to divide the hysteresis characteristics into PI model segments [18]; however, this method is based only on the physical properties of the crystal phase change of the material. No segmentation basis is associated with the operator model. When facing complex hysteresis, the 
calculation of characteristics data is large, and the increase in the number of segments will easily cause the inverse model to lose connectivity, which results in difficulty in applying compensation for complex hysteresis characteristics. Therefore, the above research is only suitable for improving the modeling accuracy of simple hysteresis characteristics. Complex hysteresis has multiple hysteresis loops, and the loops do not have the same characteristics $[19,20]$. Neither the classical PI model nor the improved PI model can eventually satisfy the requirements of uniform, high-precision compensation for complex hysteresis characteristics.

To solve the aforementioned problems, this paper proposes a Separated Level-loop PI (SLPI) model. First, the complex hysteresis characteristics data are obtained through experiments conducted under the complex working voltage of the device. Second, a loop-separation logic algorithm is used to simplify the complex hysteresis characteristics and record the loop levels and vertexes according to the characteristics change law, i.e., Madelung principle. Each single loop is modeled by the classical PI model, and the inverse model is calculated. Before compensating, a rollback method is used to restore the positive sequence of the inverse model of each inner loop of the hysteresis. The complete inverse model is reconstructed according to the vertexes and loop levels, and then used for feedforward control. Experiments show that the overall modeling accuracy of the SLPI model is higher than that of the classical PI model, and the differences in the accuracies between the loops at all levels are reduced; moreover, the compensation result for complex hysteresis characteristics is satisfactorily improved.

The remainder of this paper is structured as follows. The second section presents the design of a two-degree-of-freedom nanopositioning device based on a flexible mechanism by performing analysis and optimization; moreover, it presents a measured set of complex hysteresis characteristics data. In the third section, the characteristics of complex hysteresis data are discussed, and a reasonable 
simplification method is derived from the Madelung principle, with a detailed description of the process of the level-loop-separation logic algorithm and rollback reconstruction for feedforward control. The fourth section presents the comparison and verification experiment of the compensation control results of the SLPI and classical PI models. The fifth section presents an analogy summary of the work of this paper and similar research work.

\section{Background}

This section contains two parts. The first part describes the device that supports this paper: a nanopositioning device for precision machining based on a flexible mechanism, and its design modeling and working principles. The second part is the voltage-displacement complex nonlinear characteristic data that are measured using the positioning device in the experiment, including the detailed process and characteristic analysis.

\subsection{Mechanical design based on actuators}

The design of the nanopositioning device for precision machining refers to a type of nanopositioning stage that uses a rectangular cut-section flexure hinge [21]. The working principle of the flexible hinge mechanism is shown in Fig. 1. The expansion and contraction of the piezoelectric ceramic can be amplified by the flexible hinge and transmitted to the driven part. Therefore, the output direction of the displacement is perpendicular to the deformation direction of the piezoelectric ceramic.

The positioning device has two degrees of freedom in the $x$ and $y$ directions and supports the processing of the planar shape by the working device. During the positioning process, it is necessary to divide the specified displacement into $x$ and $y$ directions of displacement in advance to obtain the corresponding voltage value of each displacement, and then cooperate with the voltage drive output to complete the 
nanopositioning for the machining. Fig. 2 shows the three-dimensional model of the working device.

Table 1 lists the design stiffness calculation table, where $k_{x}, k_{y}, k_{z}$ are the stiffness in the $x, y$, and $z$ directions, respectively, and $k_{\theta x}, k_{\theta y}, k_{\theta z}$ are the torsional stiffness around the $x, y$, and $z$ directions, respectively [22]. The flexible hinge part adopts slow-moving wire cutting and nickel plating on the surface of the material because these processing technologies are widely used [23,24].



Fig. 1 Schematic diagram of flexible hinge 


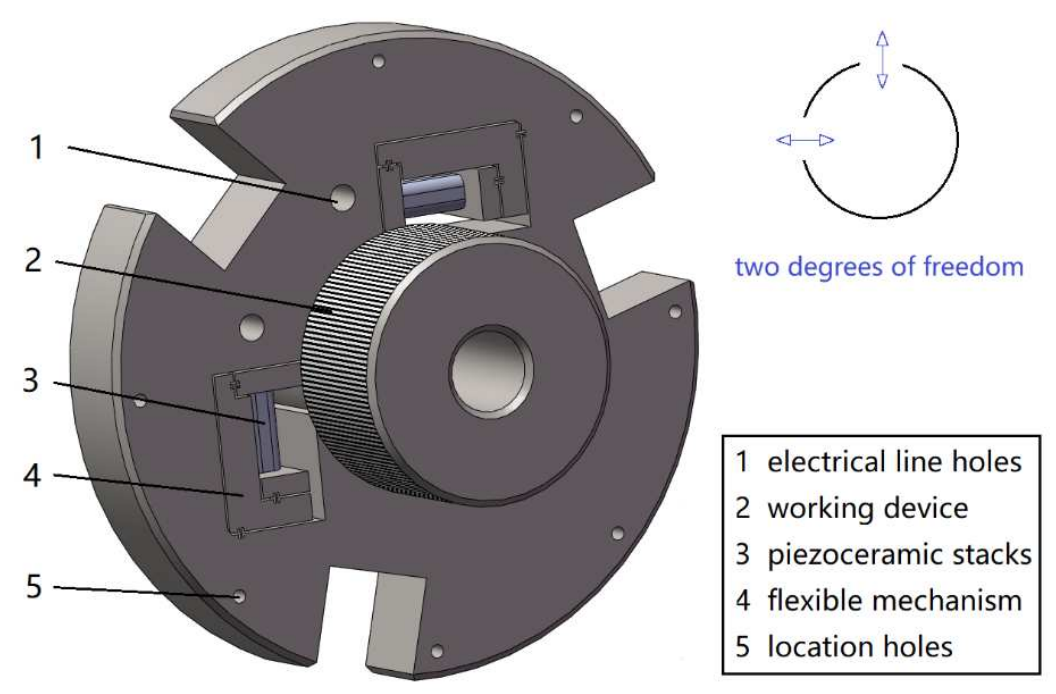

Fig. 2 Three-dimensional diagram of precision machining positioning device

Table. 1 Calculated stiffness values

$$
k_{x}, k_{y}, k_{z}: \mathrm{N} / \mathrm{mm}, k_{\theta x}, k_{\theta y}, k_{\theta z}: \mathrm{N} \cdot \mathrm{mm} / \mathrm{rad}
$$

\begin{tabular}{|c|c|c|c|c|c|c|}
\hline & $k_{x}$ & $k_{y}$ & $k_{z}$ & $k_{\theta x}$ & $k_{\theta y}$ & $k_{\theta z}$ \\
\hline Stiffness & $\approx 60$ & $\approx 10^{5}$ & $\approx 2 \times 10^{4}$ & $\approx 2 \times 10^{7}$ & $\approx 10^{8}$ & $\approx 7 \times 10^{6}$ \\
\hline
\end{tabular}




\subsection{Data acquirement and characteristic analysis}

The experimental system was built according to the equipment shown in Fig. 3. A high-precision laser interferometer manufactured by Renishaw and a 0-100 V range voltage controller were selected.

The following steps were performed to test the complex voltage-displacement nonlinear characteristics of the nanopositioning device in the $x$ direction:

(1) The computer, voltage controller, and laser interferometer were connected. The data were collected by the corresponding software of the computer. Then, the laser interferometer was adjusted to obtain a signal intensity within a reasonable range.

(2) The random complex voltage is shown in Fig. 4. The initial drive voltage was $0 \mathrm{~V}$; then, the voltage controller was used to load the electrical signal, as shown in Fig. 4.

(3) The laser interferometer was used to measure the displacement value, which was recorded once every $0.8 \mathrm{~s}$ at an interval of $4 \mathrm{~V}$. The peak and valley displacements of the complex voltage shown in Fig. 4 must be additionally recorded.

(4) The average of the two measurements was calculated. The equipment was checked and shut down.

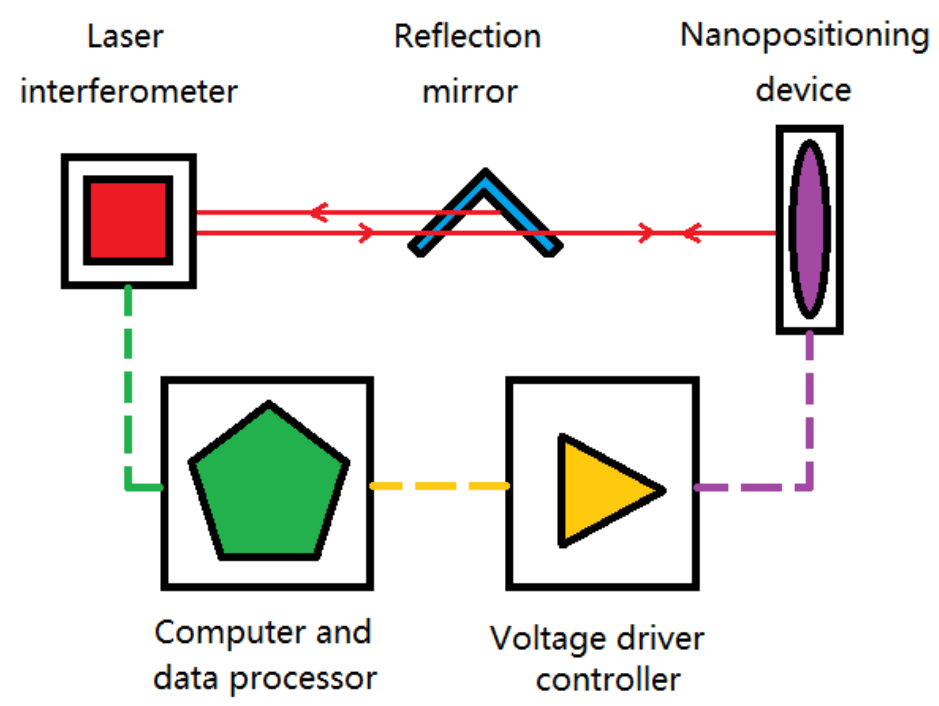

Fig. 3 Experimental system for measuring the complex characteristics 


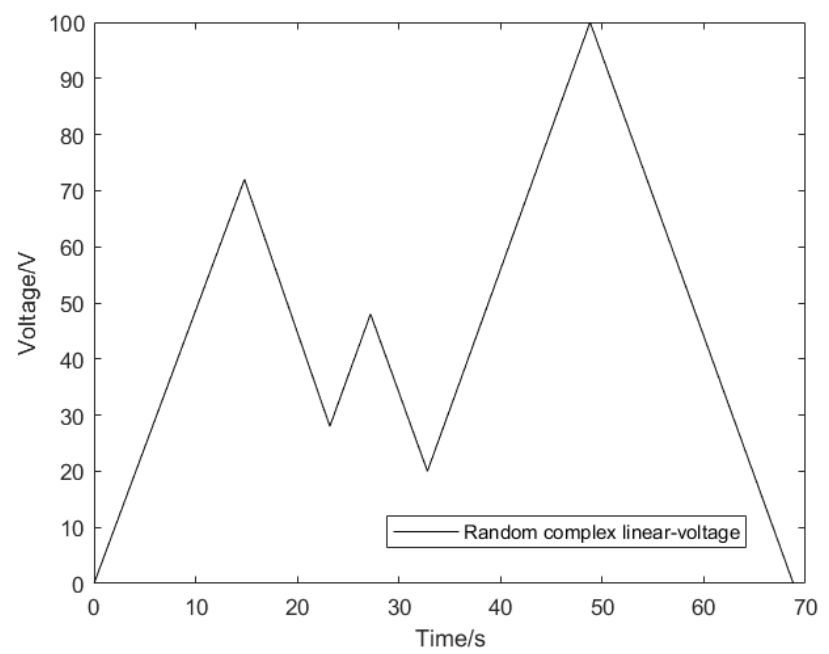

Fig. 4 Random complex voltage



Fig. 5 Nonlinear characteristics of complex voltage-displacement

The obtained nonlinear characteristics curve of the complex voltage-displacement is shown in Fig. 5.

The nonlinear characteristics of voltage-displacement of piezoelectric ceramic materials are often caused by the following three reasons [25-28]:

(1) Hysteresis characteristics: When voltage is applied to piezoelectric ceramics, the nonlinear late-achieving characteristics shown by the displacement are called hysteresis characteristics, as shown in Fig. 6a, of the voltage-displacement curve. The hysteresis of the boost phase is often shown as a concave curve, and the hysteresis of the back phase is often shown as a convex curve.

(2) Creep characteristics: After the applied voltage remains unchanged, the piezoelectric ceramic material still produces additional deformation for a period of time after the immediate response. This deformation is referred to as creep

\section{Springer}


characteristics, as shown in Fig. 6b. The rate of the extra displacement value gradually decreases with time and reaches a stable value after a period of time.

(3) Temperature-change characteristics: The property of changing the deformation ability of piezoelectric ceramic materials caused by temperature changes is called the temperature-change characteristics, as shown in Fig. 6c. Too high or too low temperature will reduce the deformability of the piezoelectric ceramics. The ideal working temperature for piezoelectric ceramic materials is between $220-390 \mathrm{~K}$.

$\mathbf{a}$
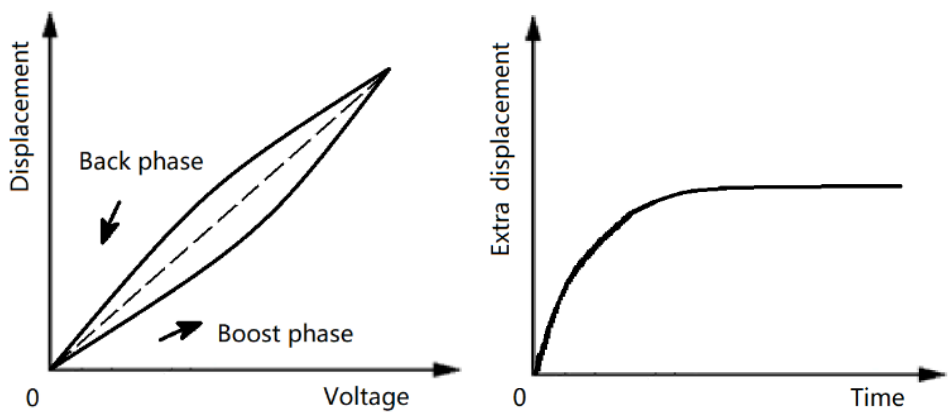

c



Fig. $\mathbf{6}$ Schematic diagram of the causes of nonlinear characteristics: a Hysteresis characteristics; $\mathbf{b}$ creep characteristics; $\mathbf{c}$ temperature characteristics

In the complex voltage-displacement curve shown in Figure 5, the nonlinear characteristics are almost all hysteresis characteristics. When outputting a complex displacement, the residence time at a specific voltage value is sufficiently short; therefore, the influence of creep characteristics can be ignored. When the working environment is at room temperature, the temperature-change characteristics are not a nonlinear factor. Therefore, the nonlinear characteristics are primarily caused by hysteresis. 
To ensure working stability, it is necessary to output a linear displacement. It is a simple and effective solution to directly analyze the nonlinear characteristics of hysteresis and use the correct mathematical model for compensation.

\section{Separated- Level-loop Prandtl-Ishlinskii (SLPI) model}

This section specifically describes the modeling ideas and steps of the SLPI model. The first part analyzes the characteristic differences of varied levels of hysteresis loops, and explains why the classical PI model cannot compensate for complex characteristics with high precision through the feature of the Play operator. The second part uses a logical algorithm for the separation to identify the vertexes of each loop and split them according to the loop level. The third part reviews the classical PI model and uses this model to describe each separated loop individually. Each loop is inverted separately after PI modeling. Therefore, the positive sequence for compensation is restored and reconstructed using a rollback method, and the total inverse model for feedforward control is finally obtained.

\subsection{Analysis of complex hysteresis characteristics and Play operator}

To establish a correct mathematical model, the complex hysteresis characteristics and PI model features are further discussed in this section.

When analyzing the characteristics of the hysteresis loop, the Madelung principle is usually used as a reference law $[29,30]$. The Madelung criterion summarizes complex hysteresis characteristics. According to one feature of the Madelung principle, only the inner loops with the same length show consistent hysteresis characteristics. However, in complex hysteresis characteristics, the probability of obtaining inner loops with the same data length is significantly low.

As shown in Fig. 7, the three hysteresis loops exhibit different hysteresis inclinations. The complex hysteresis inclination angle under the same voltage affects the modeling accuracy. 




Fig. 7 Differences in the hysteresis inclination angle of three hysteresis loops



Fig. 8 Play operator

When analyzing the features of the PI model, let us first analyze the Play operator that constitutes the PI model, as shown in Fig. 8. When the input is $x\left(t_{n}\right)$, the output Play operator expression $p\left(t_{n}\right)$ can be expressed as

$$
\left\{\begin{array}{l}
p(0)= \\
\max \left\{x(0)-r, \min \left[x(0)+r, p_{0}\right]\right\} \\
p\left(t_{n}\right)= \\
\max \left\{x\left(t_{n}\right)-r, \min \left[x\left(t_{n}\right)+r, p\left(t_{n-1}\right)\right]\right\}
\end{array},\right.
$$

where $r$ is the operator threshold, $n \in[1, e]$, and $0=t_{0}<t_{1}<t_{2}<\cdots<t_{e}$ are reasonably divided intervals. When the PI model describes the hysteresis of the inverse piezoelectric effect, the initial value of displacement is usually recorded as 0 ; therefore, $p_{0}=0$. 
When building the model, the single-sided operator in the first quadrant is selected. As shown in Fig. 8, the properties of the boost-phase single-sided operator are:

$\left\{\begin{array}{lc}p(t)=0 & x(t) \leq r \\ p(t)=x(t)-r & r<x(t) \leq x\left(t_{e}\right)\end{array}\right.$

The properties of the single-sided operator on the back phase are

$\left\{\begin{array}{l}p(t)=x\left(t_{e}\right) \quad x\left(t_{e}\right)-2 r \leq x(t)<x\left(t_{e}\right) \\ p(t)=x(t)+r \quad 0 \leq x(t)<x\left(t_{e}\right)-2 r\end{array}\right.$

It can be observed from the nature of the operator that, when compared to the classical PI model, the generalized or segmented improved PI models show an increase in accuracy when describing the hysteresis loop under simple voltage; however, these models do not perform well while considering complex hysteresis characteristics with multiple inner loops. There are two primary reasons: one is that complex hysteresis data tends to have uneven distribution of hysteresis characteristics; therefore, large and small data segments are not suitable for building the optimal model under the same number of iterations; the second is that the model itself increases parameters; therefore, when facing complex hysteresis characteristics, the generalized improved model is embodied in additional mathematical formulas that continue to be superimposed and nested, and the segmented improvement is embodied in too many segmented points. The influence of a large number of redundant parameter problems and segmental cohesion problems on compensation control cannot be ignored. In addition, the generalized and segmented improved PI model also has other problems, such as a significant increase in computation.

The modeling essence of the PI model is the weighted superposition of a limited number of Play operators. From the perspective of modeling features, when describing a single hysteresis loop, the boost-phase operator is selected for the data increase edge, and the back-phase operator is selected for the data reduction edge, which can model the hysteresis loop with high accuracy without affecting each other. From the perspective of hysteresis characteristics, a complex hysteresis often has multiloop characteristics. Multiple single loops of different lengths contained in multiple loops corresponding to different hysteresis inclination angles. There are certain voltage values corresponding to more than 
one unilateral displacement value. Hence, the modeling accuracies of each segment are affected. Judging from the above two perspectives, to eliminate the influence of operator modeling, different hysteresis loops should be modeled strictly according to their independent characteristics. Therefore, a single hysteresis level-loop-separation logic algorithm is proposed.

\subsection{Logical algorithm for level-loop separation}

The Madelung principle summarizes the characteristics of the complex hysteresis loop. Except for the law mentioned in the first part of this section, there are three more important laws:

(1) Turning point law: The characteristic of the hysteresis curve starting from the loop turning point is uniquely determined by this point.

(2) Loop formation law: The trend of the hysteresis curve starting from a new turning point tends to return to the last turning point.

(3) Regression law: The trend of the curve returns to the last lower-level loop after formation and exceeds the last turning point constructed by law (2)

It can be determined from the above laws that the complex hysteresis characteristics must be composed of several hysteresis inner loops and one hysteresis outer loop. To eliminate the influence of the Play operator modeling in the PI model, single hysteresis loops should be separated. The outer loop is defined as the zero-level loop, and the number of levels gradually increases; therefore, the innermost hysteresis ring has the highest level. In the separation process, the priority of separating the data of the high-level loop does not affect the hysteresis characteristics of the low-level loop. To achieve level-loop separation, it is necessary to obtain the vertex information and loop-level information from the complex hysteresis characteristics.

Hence, a logical algorithm of single level-loop separation is used to divide the complex characteristics into single hysteresis loops at all levels, including the number of loop levels and vertex information. To facilitate the calculation of the algorithm, the $k$ th experimental data is converted from ( voltage $_{k}$, displacement $_{k}$ ) into the following format:

$$
\operatorname{NewData}(k):\left[k, v_{k}, d_{k}, \operatorname{tr}(k), \operatorname{lv}(k), \operatorname{sp}(k)\right],(4)
$$


where $\mathrm{k}$ is the measurement sequence when acquiring data, $v_{k}$ and $d_{k}$ are the $k$ th voltage data and corresponding displacement data, respectively, $\operatorname{tr}(k)$ is the mark of the boost or back phase: $\operatorname{tr}(k)=1$ indicates the boost phase and $\operatorname{tr}(k)=-1$ indicates the back phase, $\operatorname{lv}(k)$ is the value of the hysteresis loop level of the $k$ th data, $s p(k)$ is the vertex identifier: the non-vertex data $s p(k)=$ 0 , the suspected vertex data $s p(k)=1$, and the true vertex data $s p(k)=2$. The logical judgment process is started from the initial data in the data sequence. The outer-loop level is the zero level, and the initial phase is the boost phase. The zero point must be the true vertex of the outer loop or the zero-level loop; hence, the zero point is defined as

$$
\operatorname{NewData}(0)=[0,0,0,1,0,2] .
$$

After determining the initial new data, the subsequent data are judged sequentially. Because of the data changes at the turning points of the hysteresis curve, the sequence trend must be increased and decreased alternately; therefore, the logic algorithm flow is also an alternate cycle of boost-back logic judgment. When the $k$ th data is not a turning point in the hysteresis curve, it satisfies the following condition.

$$
\left\{\begin{array}{lr}
v_{k+1}-v_{k}>0 & \operatorname{tr}(k)=1 \\
v_{k+1}-v_{k}<0 & \operatorname{tr}(k)=-1
\end{array}\right.
$$

At this time, it is impossible for the $k$ th data to be a vertex; therefore, $s p(k)=0$. The level value of subsequent data hysteresis loops remains unchanged: $\operatorname{lv}(k)=$ $l v(k-1)$; moreover, the boost-back mark remains unchanged: $\operatorname{tr}(k)=\operatorname{tr}(k-$ 1).

The decreasing data appearing in the boost phase and the increasing data appearing in the back phase are the turning points of the hysteresis curve. When the limited influence of creep characteristics on the data is ignored, a turning point satisfies the following condition.

$$
\left\{\begin{array}{lr}
v_{k+1}-v_{k}<0 & \operatorname{tr}(k)=1 \\
v_{k+1}-v_{k}>0 & \operatorname{tr}(k)=-1
\end{array}\right.
$$

The Madelung principle stipulates that two turning points form a closed loop, and one of them is the vertex of the closed loop. Therefore, when a new turning point NewData $\left(k_{s}\right)$ is determined by Eq. (7), the current data should be marked as a

\section{Springer}


suspected vertex: $s p(k)=1$. Then, one is added to the value of the hysteresis loop level for the data: $l v(k)=l v(k-1)+1$, and the boost-back mark is inverted: $\operatorname{tr}(k)=-\operatorname{tr}(k-1)$. The suspected vertex must use the subsequent logic to determine whether the uncertain vertex is removed from the suspicion or becomes a true vertex. The authenticity of suspected vertexes is judged in the following situations:

(1) When the logic judgment flow is in the back phase, i.e., $\operatorname{tr}(k)=-1$, if the voltage data value is judged to be less than or equal to the last suspected vertex NewData $\left(k_{a}\right)$, whose value of level is the current level minus one, then confirm that $\operatorname{sp}\left(k_{a}\right)=2$;

(2) When the logic judgment flow is in the boost phase, i.e., $\operatorname{tr}(k)=1$, if the voltage data value is judged to be greater than or equal to the last suspected vertex NewData $\left(k_{a}\right)$, whose value of level is the current level minus one, then confirm that $\operatorname{sp}\left(k_{a}\right)=2$;

After confirming that $N e w D a t a\left(k_{a}\right)$ is the true vertex with the hysteresis loop level of $l v\left(k_{a}\right)=\operatorname{lv}(k)-1$, all the data of this level are separated under the hysteresis single loop starting and ending from the vertex. The separated data are sewed at the vertex to form a single hysteresis loop with level value and vertex information. Then, $l v(k+1)=l v(k)-2$, and subsequent judgments are determined.

If the separation of the hysteresis loop is performed on a higher-level loop before the new separation, separation is performed on the remaining data after the last separation. The remaining data are sewed together at the vertex of the previously separated single hysteresis loop to form a new hysteresis characteristic waiting for the next separation. 
$\mathbf{a}$

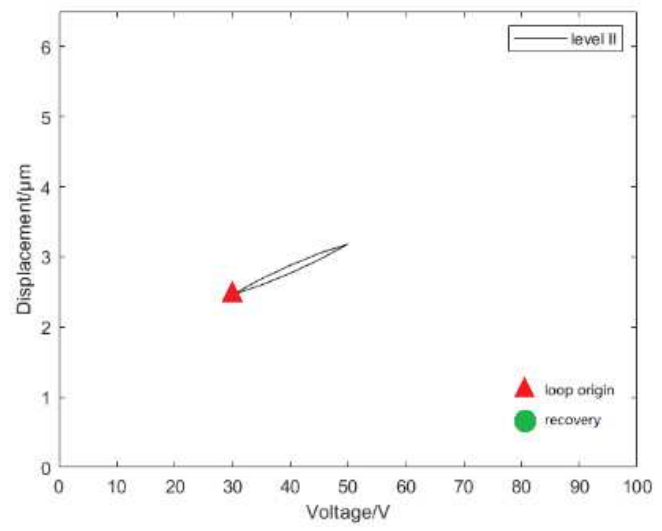

b

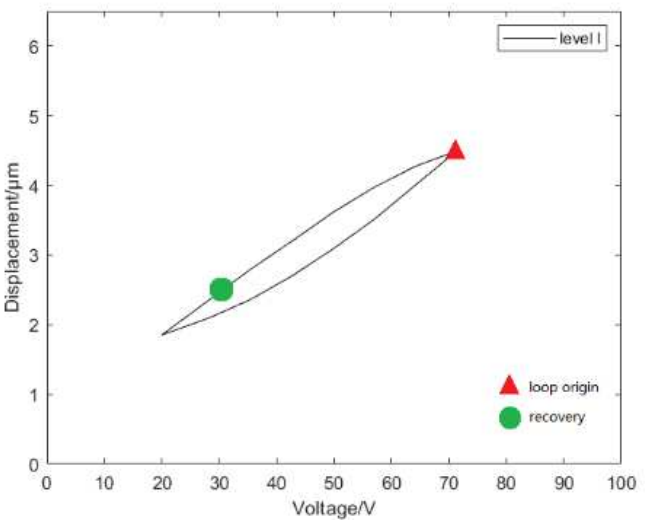

c

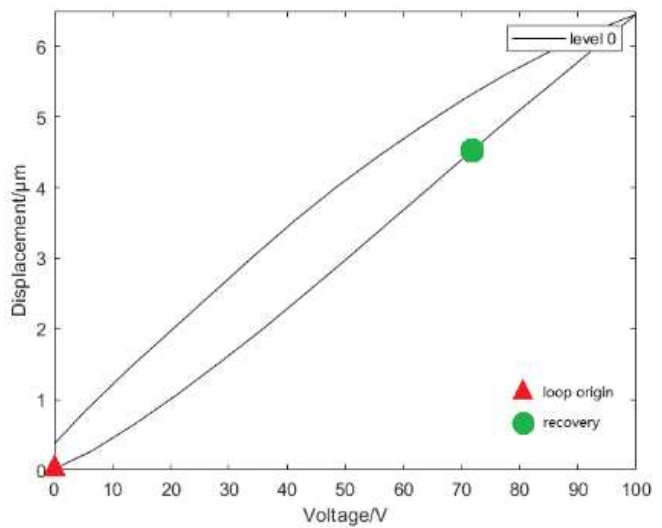

Fig. 9 Single-loop-separation results: a Level-II single loop; b level-I single loop; c level-0 single loop

After operating the single-loop-separation logic algorithm, the original complex multiloop hysteresis characteristics are separated into several hysteresis single loops of different levels from the highest value to zero. Fig. 9 shows three hysteresis single-loop images obtained from the data in Fig. 5 after applying the single-loop separation logic algorithm. The triangle signs mark the vertexes as original points of the loop of each level of the hysteresis loops, and the circle signs mark the position of the sewed point as a recovery of the lower hysteresis loop after the higher one is separated. Fig. 9a shows the highest-level inner loop in the experimental data; therefore, this single hysteresis loop is preferentially separated from the level-II true vertex. Fig. 9b shows the level-I hysteresis loop. The data are separated from the level-I true vertex and sewed at the level-II true 
vertex. Fig. 9c shows the remaining level-0 outer hysteresis loop; therefore, the true vertex of the outer loop is the zero point.

In a period of complex hysteresis with nonlinear characteristics, the largest data of the curve, which is also a turning point, will be defined as a suspected level-I vertex when it is detected. Therefore, suspected vertexes that appear on the back phase of the outer loop will continue to increase the level value while the original suspected vertex still exists, until the judging process is completed. The phenomenon of adding one to the level value in the back phase will not affect the result of the single-loop-separation logic algorithm because the purpose of the logic algorithm is to separate the hysteresis single loop, and the separation step depends only on the level values and position of the true vertexes. Consequently, the exact number of level values is irrelevant.

In conclusion, the single-loop-separation logic algorithm imports the data in the order measured in the experiment and applies the sequential conditional sentence detection algorithm of the programming language. Logical judgment filters out the hysteresis loop vertexes with loop-value information. Then, the obtained levelvalue information is used for separation from the highest value to zero. In the next two parts of this section, high-precision modeling of complex hysteresis characteristics can be obtained by using the PI modeling on each single loop.

\subsection{Prandtl-Ishlinskii and inverse models}

Each obtained single-loop hysteresis is modeled independently by the PI model. As shown in Fig. 8, unilateral operators are selected for the boost and back phases. The selected operators are weighted and superimposed. The PI model can be expressed as

$$
P[x(t)]=\omega_{0} \cdot x(t)+\sum_{i=1}^{n} \omega_{i} \cdot p_{i}(t),
$$

where $P[x(t)]$ is the PI model output corresponding to the operator input $x(t)$. $\omega_{0}$ is the first weight, which is usually a positive number. The corresponding output of the $i$ th operator is $p_{i}(t)$, which has a corresponding threshold $r_{i}$ and corresponding weight $\omega_{i}$. 
Fig. 10 is a modeling diagram of three single hysteresis loops obtained by the separation method using the PI model. It can be observed from the modeling results that the PI model has high modeling accuracy for each hysteresis single loop with different hysteresis inclination angles, which verifies that the theory of eliminating the overlapping effects of operator features is feasible.

When modeling the hysteresis, as shown in Fig. 10, a quadratic programming iteration is used to determine the optimal parameter values. In this algorithm, the minimum number of iterations required to obtain the optimal modeling for each single loop is different. For a single loop of length $m$, the minimum iterations $A_{m}$ are usually considered as

$$
A_{m}=10 \sqrt{\frac{4 m}{m_{e}}+1}
$$

where $m_{e}$ is the total length of the experimental data.

a

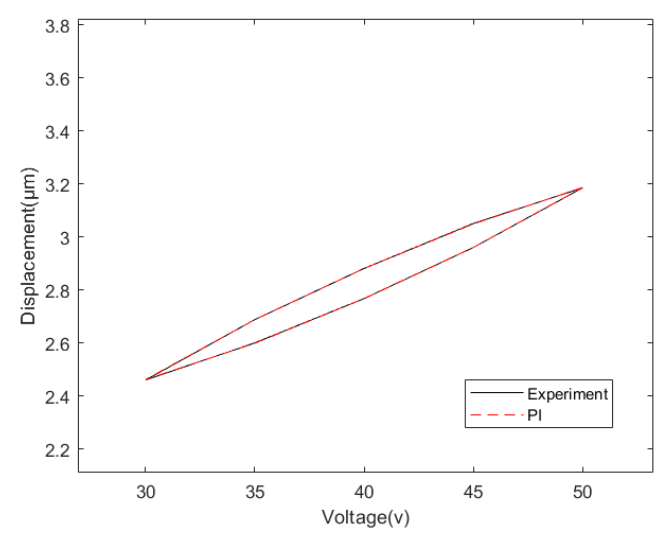

b

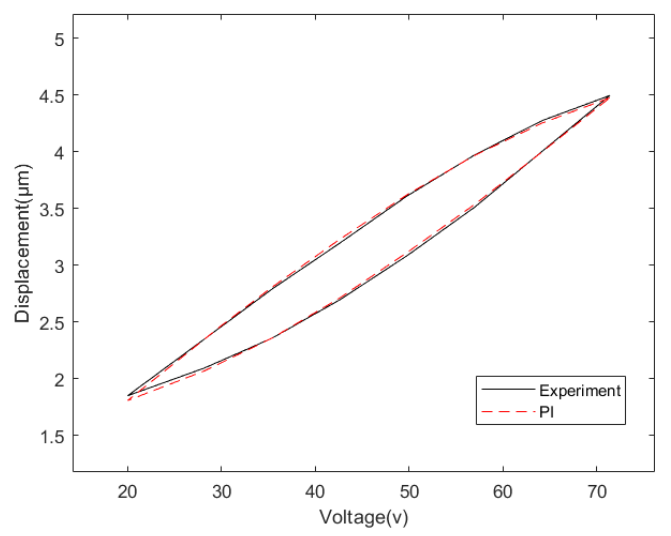

c

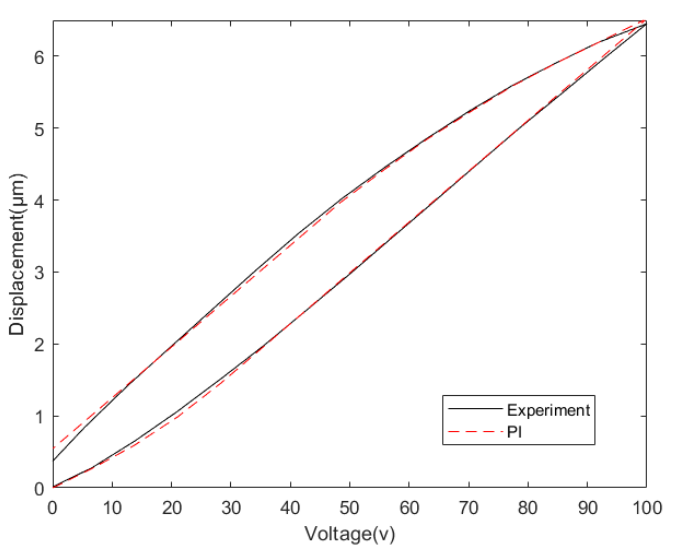


Fig. 10 Single-loop Prandtl-Ishlinskii (PI) modeling: a Level-II single-loop modeling; b Level-I single-loop modeling; c Level-0 single-loop modeling

Tables 2, 3, and 4 list the parameters of Figs. 10a, 10b, and 10c, respectively. The threshold $\boldsymbol{r}_{\boldsymbol{i}}$ and optimal weights $\boldsymbol{\omega}_{\boldsymbol{i}}$ are listed in the tables.

Table. 2 Parameter values of Fig. 10a

\begin{tabular}{ccc}
\hline $\boldsymbol{i}$ & $\boldsymbol{r}_{\boldsymbol{i}}$ & $\boldsymbol{\omega}_{\boldsymbol{i}}$ \\
\hline 1 & 0 & 0.02943 \\
2 & 5 & 0.01351 \\
3 & 10 & 0.00040 \\
4 & 15 & 0.00206 \\
5 & 20 & 0.00230 \\
6 & 25 & 0.00265 \\
7 & 30 & 0.00249 \\
8 & 35 & 0.00550 \\
9 & 40 & 0.02802 \\
\hline 10 & 45 & 0.09411 \\
\hline
\end{tabular}

Table. 3 Parameter values of Fig. 10b

\begin{tabular}{ccc}
\hline $\boldsymbol{i}$ & $\boldsymbol{r}_{\boldsymbol{i}}$ & $\boldsymbol{\omega}_{\boldsymbol{i}}$ \\
\hline 1 & 0 & 0.03151 \\
2 & 5 & 0.01298 \\
3 & 10 & 0.01092 \\
4 & 15 & 0.00445 \\
5 & 20 & 0.00648 \\
6 & 25 & 0.00000 \\
7 & 30 & 0.00148 \\
8 & 35 & 0.00016 \\
\hline
\end{tabular}




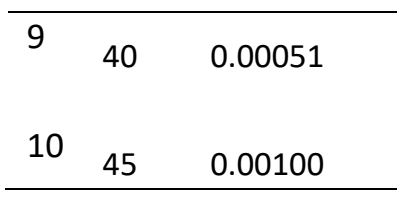

Table. 4 Parameter values of Fig. 10c

\begin{tabular}{ccc}
\hline $\boldsymbol{i}$ & $\boldsymbol{r}_{\boldsymbol{i}}$ & $\boldsymbol{\omega}_{\boldsymbol{i}}$ \\
\hline 1 & 0 & 0.04045 \\
2 & 5 & 0.00000 \\
3 & 10 & 0.00893 \\
4 & 15 & 0.00537 \\
5 & 20 & 0.00456 \\
6 & 25 & 0.01136 \\
7 & 30 & 0.00000 \\
8 & 35 & 0.00000 \\
9 & 40 & 0.00000 \\
10 & 45 & 0.00000 \\
\hline
\end{tabular}

In the SLPI model, the minimum number of iterations can be selected according to the length of the separated data so that the modeling of each hysteretic single loop can achieve the highest accuracy as well as avoid redundant calculations. However, the PI model for the complete complex hysteresis characteristics does not have the existence condition of the minimum iterations; consequently, a uniform number of iterations leads to modeling error.

The PI model has an analytical inverse model. The SLPI model is composed of a combination of independent single-loop PI models. Therefore, the inverse model equation of each separated single loop is consistent with the classical PI inverse model. The formula is expressed as

$$
\begin{gathered}
\mathrm{P}^{-1}[p(t)]=\omega_{0}^{-1} \cdot p(t)+\sum_{i=1}^{n} \omega_{i}^{-1} \cdot x_{i}(t),(10) \\
x_{i}(t)=\max \left\{p(t)-r_{i}^{-1}, \min \left[p(t)+r_{i}^{-1}, x_{i-1}(t)\right]\right\},(11)
\end{gathered}
$$

where $\mathrm{P}^{-1}[p(t)]$ is the output corresponding to the input $p(t)$ of the classical PI inverse model operator and $x_{i}(t)$ is the output of the $i$ th operator. To

\section{Springer}


correspond to the PI-positive model, $x_{0}=0$. The threshold $r_{i}^{-1}$ and weight coefficients $\omega_{i}^{-1}$ of the PI inverse model are expressed as follows.

$$
\left\{\begin{array}{c}
r_{i}^{-1}=\sum_{j=1}^{i} \omega_{j} \cdot\left(r_{i}-r_{j}\right) \\
\omega_{i}^{-1}=-\frac{\omega_{0}^{-1}=\frac{1}{\omega_{0}}}{\left(\omega_{0}+\sum_{j=1}^{i-1} \omega_{j}\right)\left(\omega_{0}+\sum_{j=1}^{i} \omega_{j}\right)}
\end{array}\right.
$$

Based on the handling of each single-loop inverse model, a complete SLPI inverse model can be established, and the feedforward control method can be used to compensate for the complex hysteresis characteristics.

\subsection{Compensation theory and inverse SLPI model}

The method of feedforward control collects information through a pre-experiment, observes the law to estimate the trend, and applies pre-control methods in front of the system to correct the compensation result. It is one of the commonly used compensation control methods in control engineering [31-33].

The nonlinear hysteresis characteristics shown by the voltage-displacement curve of the inverse piezoelectric effect follow the Madelung principle. There is no occasional variability and no requirement for feedback in the control. The PI model used in the modeling is a phenomenological model that describes the characteristics directly without explaining the internal reasons. Therefore, feedforward control is the most effective compensation method for the piezoelectric complex hysteresis characteristics. A schematic diagram of the feedforward compensation control is shown in Fig. 11.

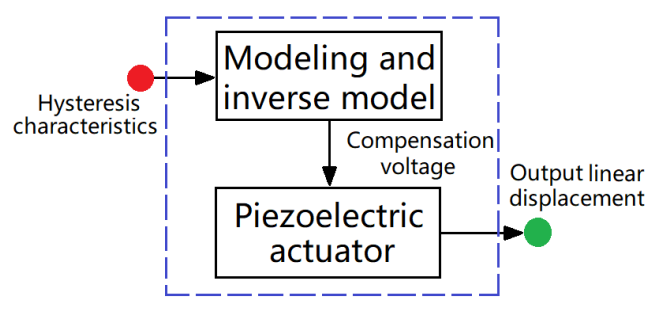

Fig. 11 Schematic diagram of feedforward compensation control

It can be observed from Fig. 11 that the PI model of the hysteresis single loops should be restructured to construct the SLPI inverse model for the feedforward 
control compensation voltage. After obtaining the PI model of single loops, the reconstruction steps are as follows:

(1) Single-loop model inversion: The analytical inverse model of each hysteretic single loop is obtained through the PI inverse-model equation. The level value information of the hysteretic single loop is retained, as well as the true-vertexmodeling and sewed-connection-point positions.

(2) Single-loop inverse model restoration: The sewed point on a single hysteretic loop is the vertex of a higher-level hysteresis loop starting from this loop. The inverse model of the hysteresis single loop must be clockwise. Therefore, starting from the level-0 outer loop, the sewed point on the current hysteresis loop is changed into a break point. The inverse model of the current loop is divided into two parts. The outer-loop inverse model is linked to the inverse model of the new loop at the break position of the inverse model of the level-I inner loop. The new inverse model sequence is obtained in two loops that have two clockwise directions. This step is repeated to reconstruct the higher-level hysteresis single loop until all the inverse models of the single inner loops are connected at break points. Finally, all the inverse model restorations are completed. In the sequence restoration process, the vertexes or break points of the same level should be divided and spliced sequentially in the order of initial separation level value, without affecting the reconstruction result of the achieved inverse model restoration.

Fig. 12 shows the inverse model corresponding to the three hysteresis single-loop models in Fig. 10. The star sign is the position of the break point on the level-I and level-0 inverse models. Fig. 13 shows the inverse model of SLPI after sorting and reconstruction according to the restoration method described above. The SLPI inverse model is the displacement-voltage correspondence curve. The obtained compensation voltage can be directly used for feedforward control. The compensation result of the feedforward control must be further verified through experiments and discussion. 

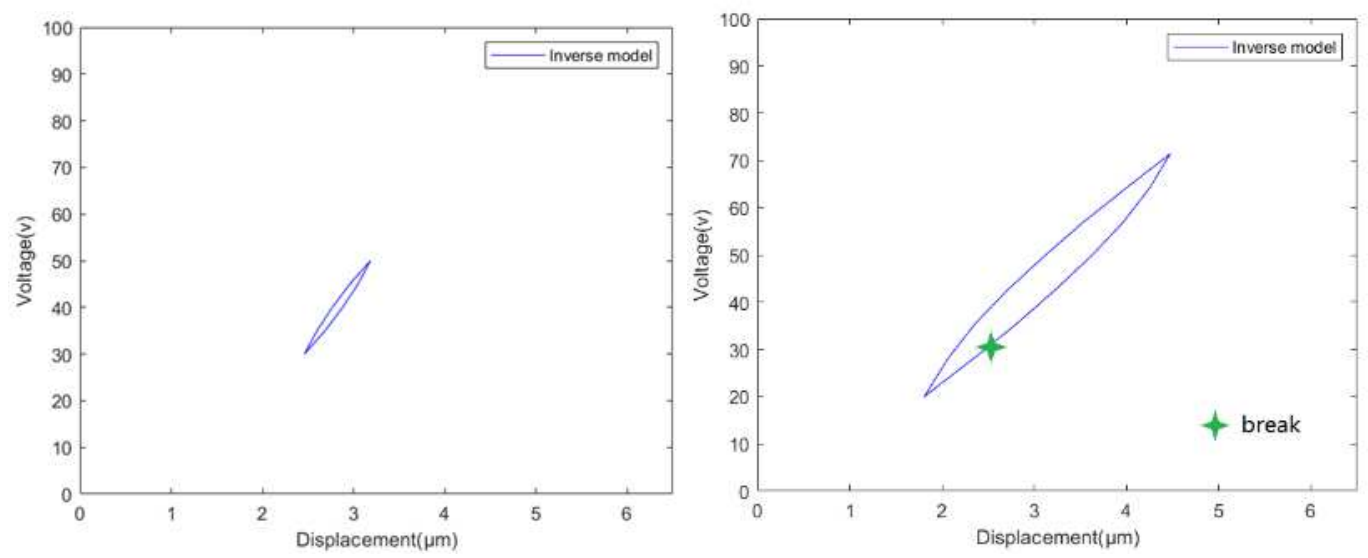

c

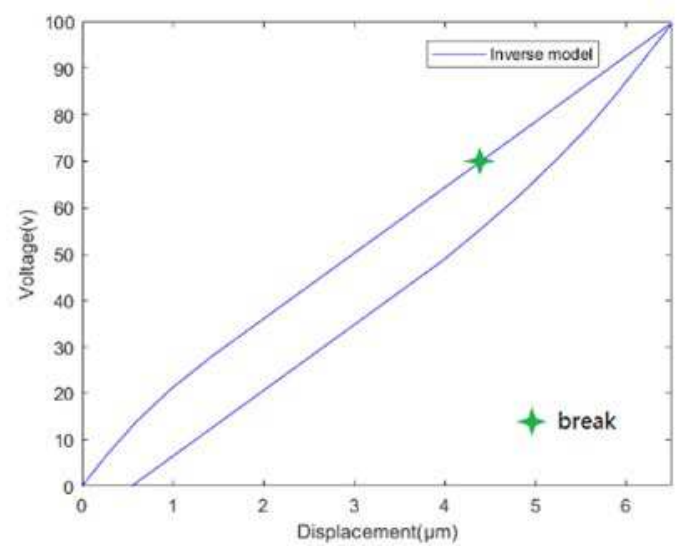

Fig. 12 Single-loop inverse model: a Level-II single-loop inverse model; b level-I single-loop inverse model; c level-0 single-loop inverse model



Fig. 13 Separated Level-loop PI (SLPI) inverse model 


\section{Experiment and discussion}

This section presents the experimental verification and discussion on the results.

Two fundamental comparisons are shown in the diagrams. One is a comparison of the modeling description of the classical PI and SLPI models, and the other a comparison of the final compensation results.

In addition, this section adds two additional sets of special verification experiments. One is to verify whether the inner loop starting from the back-phase hysteresis characteristics causes errors in the modeling. The other is to verify whether the overlapped outer-loop data affects the compensation method.

\subsection{Model comparisons}

The modeling description determines the compensation result. Observing the modeling error between different models and the actual hysteresis characteristics curve can directly reflect the comparison of accuracy. The modeling of the classical PI model is shown in Fig. 14a. The modeling of the three hysteretic single loops is drawn on the same diagram as SLPI modeling, as shown in Fig. $14 \mathrm{~b}$.

$\mathbf{a}$

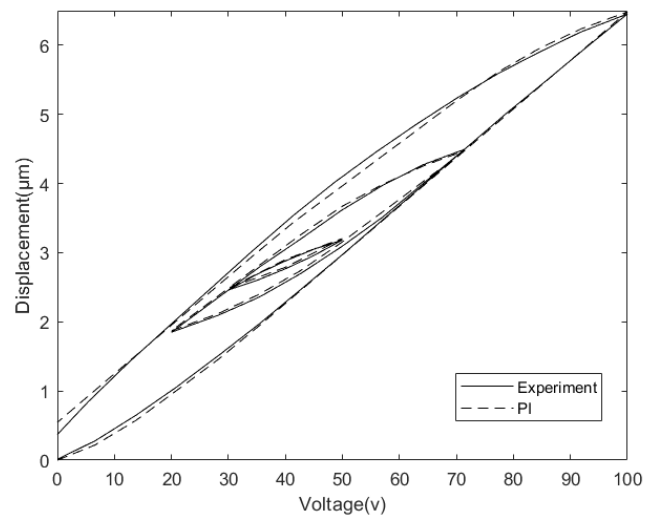

b



Fig. 14 Modeling comparison diagrams: a Classical PI model comparison diagram; b gathered SLPI model comparison diagram

Table 5 lists the parameters of the model shown in Fig. 14a. The threshold $\boldsymbol{r}_{\boldsymbol{i}}$ and optimal weights $\boldsymbol{\omega}_{\boldsymbol{i}}$ are listed in the table.

Table. 5 Parameter values of model shown in Fig. 14a 


\begin{tabular}{rrr}
\hline $\boldsymbol{i}$ & $\boldsymbol{r}_{\boldsymbol{i}}$ & $\boldsymbol{\omega}_{\boldsymbol{i}}$ \\
\hline 1 & 0 & 0.03040 \\
2 & 5 & 0.01116 \\
3 & 10 & 0.01516 \\
4 & 15 & 0.00478 \\
5 & 20 & 0.00000 \\
6 & 25 & 0.00000 \\
7 & 30 & 0.00823 \\
8 & 35 & 0.00057 \\
9 & 40 & 0.00000 \\
10 & 45 & 0.00000 \\
\hline
\end{tabular}

By comparing the modeling diagrams shown in Fig. 14, it can be observed that the SLPI model has a higher modeling accuracy and shows the adaptive features of the material-based characteristics of the outer loop. In contrast to the phenomenon where the accuracy deteriorates in higher-level loop modeling, the SLPI model always maintained the accuracy.

To verify the comparison of the actual compensation results, experiments were performed using the compensation voltage of both the classical PI and SLPI inverse models as follows:

(1) Experimental equipment such as piezoelectric driver, laser interferometer, and reflector were placed on the horizontal test bench and connected with a voltage driver and computer.

(2) The compensation voltage obtained from the classical PI inverse model was loaded through the controller at an ordinary control speed and the displacement was recorded. Similarly, the compensation voltage obtained from the SLPI inverse model was loaded at the ordinary control speed and the displacement of the experiment was recorded.

(3) The control speed of voltage loading was reduced and displacements for the two model compensation results were recorded again. 
(4) The displacement data were analyzed and the average value of the actual displacement output was calculated according to the compensation results of the different models measured above.

(5) The equipment was checked and switched off.

Owing to the limited influence produced by the creep characteristics, the experiment used the measurement method of changing the compensation control speed to obtain the average value of the compensation result. The comparison results are as follows. Fig. 15a illustrates the compensation result curve of the classical PI inverse model and Fig. 15b shows the compensation result of the SLPI inverse model under compensation voltage.

a

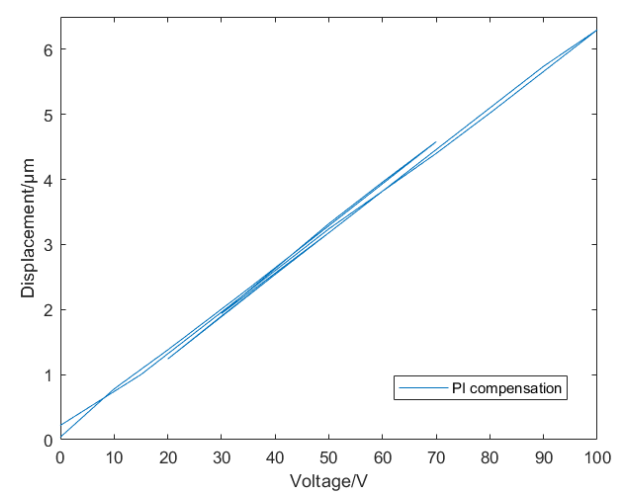

c

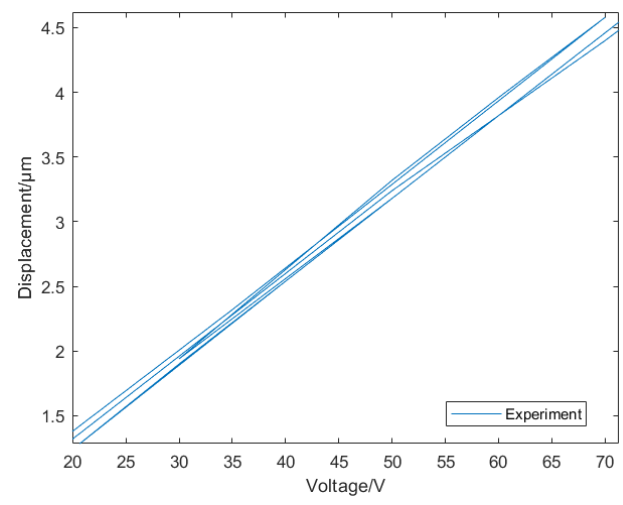

b

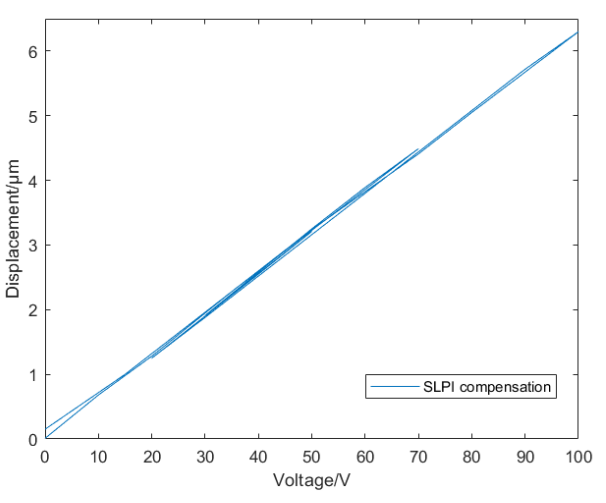

d

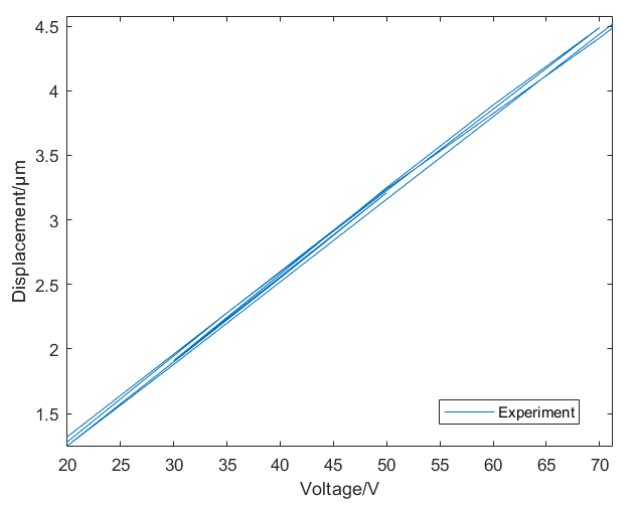


Fig. 15 Compensation result diagrams: a Classical PI compensation, b SLPI compensation, c partially enlarged Fig. 15a, d partially enlarged Fig. 15b

By considering the value on the straight line passing through the zero point and the maximum value of the experimental data as the expected linear output values, the compensation error is the difference between the compensation result and the expected result. The error $\varepsilon$ and average error $\bar{\varepsilon}$ are

$$
\begin{aligned}
& \varepsilon_{\tau}=\left|\delta_{\tau}-c_{\tau}\right|, \\
& \bar{\varepsilon}=\frac{1}{\lambda} \sum_{\tau=1}^{\lambda}\left(\left|\delta_{\tau}-c_{\tau}\right|\right),
\end{aligned}
$$

where $\lambda$ is the total number of experimental samples for the compensation control, $\tau \epsilon[0, \lambda] . \delta_{\tau}$ is the expected displacement output and $c_{\tau}$ is the real displacement output.

As predicted by the comparative modeling description, the compensation results of the SLPI inverse model are better than those of the classical PI model. They are specifically embodied as follows:

(1) Improvement of inner-loop compensation: The compensation effect of highlevel inner-loop hysteresis is significantly improved, and it effectively solves the problem where the higher-level loop has lower accuracy in the complex hysteresis characteristics. Meanwhile, the problem of the obvious compensation error difference between hysteresis loops is also corrected.

(2) Stability of the outer-loop compensation: Based on the compensation of the SLPI model, the compensation results based on the material-based characteristics for the compensation of the outer loop do not show lower accuracy than the classical PI model. The error of the SLPI model is even smaller and the accuracy is higher.

(3) Self-elimination of compensation error: When using the SLPI inverse model for hysteresis compensation, the cumulative and new errors will erase each other more often than they overlap each other. This increase in accuracy may occur accidentally due to the position of the vertex of the hysteresis loop, but it does not deny the possibility that the single-loop modeling itself may eliminate the 
asymmetrical features, which is a phenomenon beneficial to compensation control.

In the calculation result of the percentage index, the SLPI inverse model compensation reduces the error of maximum difference value on the outer loop by $10.0 \%$, and remarkably reduces the average error of the highest-level inner loop by $97.5 \%$. Although there is an unpredictable small error increase on a vertex of the level-I loop, the error difference between the single hysteresis loops is reduced by $53.7 \%$ when compared to the classical PI inverse model, and the overall average error is significantly reduced by $50.3 \%$.

\subsection{Other verifications}

Past experiments on the inverse piezoelectric effect show that external disturbances such as the frequency of the control voltage rarely affect the application of the PI model. Therefore, whether the compensation of the SLPI inverse model has a superior effect depends on the characteristics of its own modeling; consequently, two sets of verification experiments are designed. The first experiment used the double inner-loop hysteresis data of a nanopositioning stage device, as shown in Fig. 16. It can be observed from the data that one of the true vertices is located on the back phase of the outer loop. It can be verified by observing the modeling diagram whether the hysteresis characteristics starting from the back phase of the outer loop can also be compensated by the SLPI model.

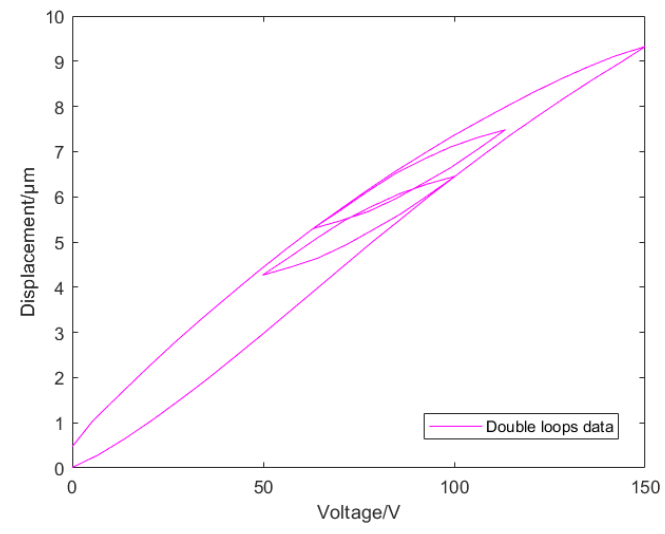

Fig. 16 Double inner-loop hysteresis data

Fig. 17a shows the optimal modeling diagram of the classical PI model of double inner-loop hysteresis data. It can be observed that the classical PI model will share

\section{Springer}


the error on each hysteresis loop to maintain the overall optimal modeling, which results in a large number of low-fitting models. This phenomenon is more obvious in the case of multiple inner loops, especially when there are vertexes for both the boost and back phases. Fig. $17 \mathrm{~b}$ is a gathered diagram of each hysteresis singleloop model in the SLPI model. Although the description of material-based nonlinear characteristics cannot be improved by single outer-loop modeling, the influence of the vertex on the back phase is almost eliminated.

a

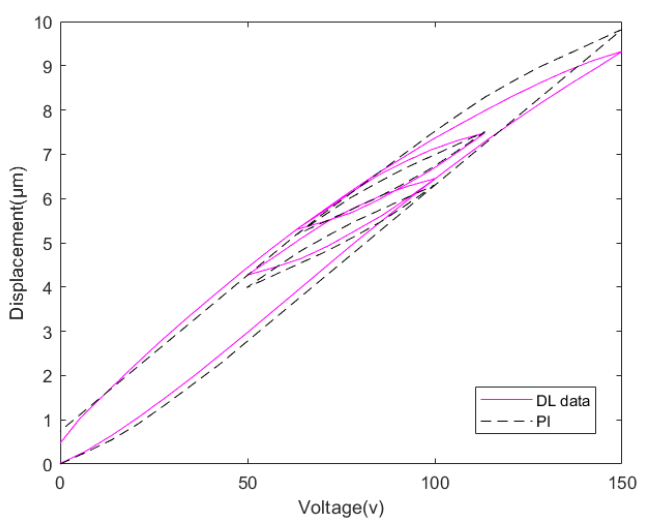

b

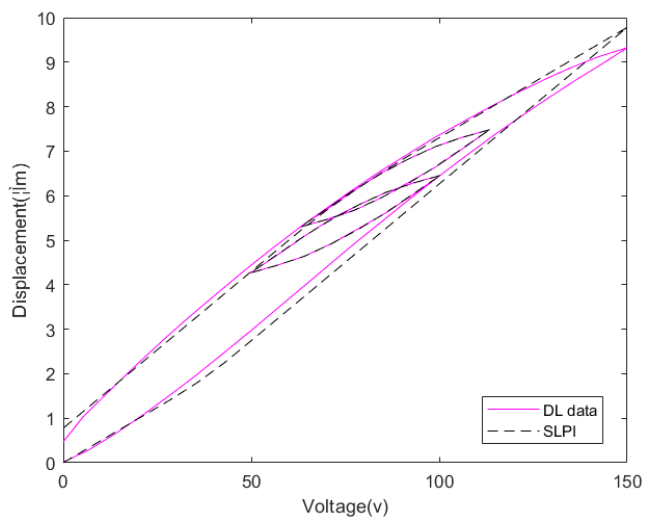

Fig. 17 Comparison of double inner-loop hysteresis modeling: a Classical PI modeling; b SLPI modeling



Fig. 18 Overlapped hysteresis data

The second set of experiments obtains the hysteresis data of another piezoelectric actuator device, as shown in Fig. 18. It can be observed from the diagram that there are overlapped data of the inner and the outer hysteresis loops. Therefore, it is verified by modeling whether the repeated hysteresis characteristics on different hysteresis single loops are also compensated well by the SLPI model. 
$\mathbf{a}$

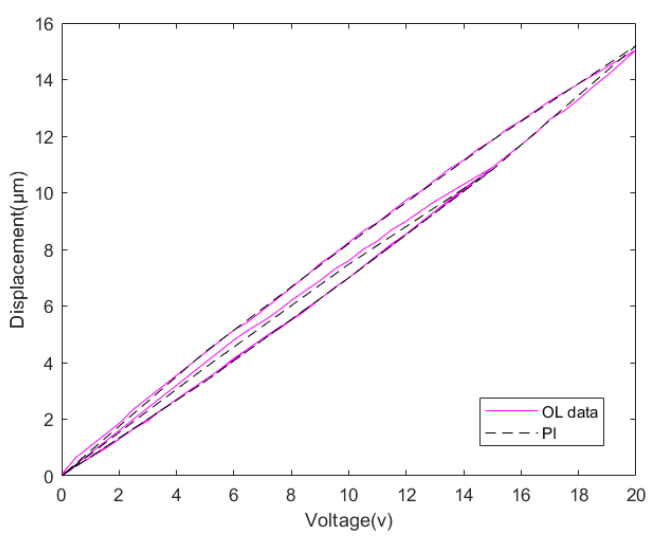

b

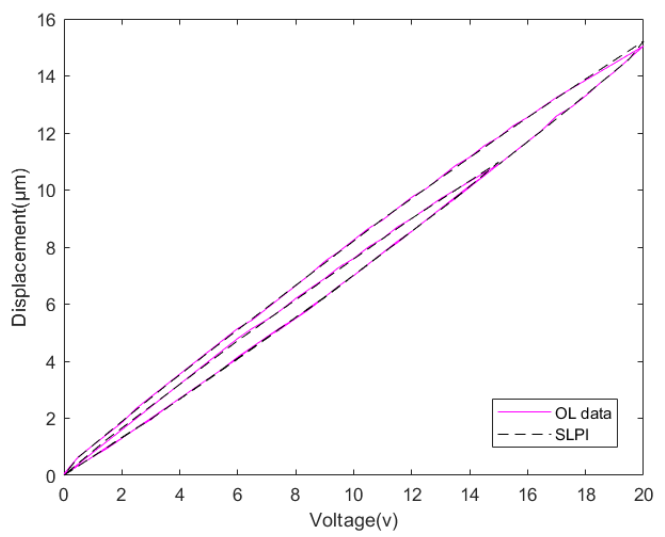

Fig. 19 Comparison of overlapped hysteresis modeling: a Classical PI modeling; b SLPI modeling Fig. 19a shows the optimal modeling diagram of the classical PI model and Fig. $19 \mathrm{~b}$ illustrates the gathered diagram of each hysteresis single-loop modeling performed by the SLPI model. Through comparisons, it can be observed that the two single-loop models are not affected by the overlapped outer-loop hysteresis. The maximum errors of the classical PI model still appear in the inner loop, and the SLPI model effectively corrects this error.

In conclusion, in the first set of verification experiments, the modeling error difference between the two inner loops of the SLPI model is smaller than that of the classical PI model, and both loops have high accuracy. When compared to the large error jump of classical PI modeling, the SLPI modeling of the materialbased characteristics of the outer loop is closer to the nonlinear curve; hence, the overall accuracy is also improved. In the second set of control experiments, the SLPI modeling is not affected by repeated hysteresis characteristics. The modeling description of the SLPI model is better. Therefore, the SLPI model has a high fitting accuracy for all types of complex hysteresis characteristics, and the improved modeling accuracy indirectly supports the enhancement in compensation control.

\section{Conclusion}

The SLPI model generally improves the compensation control accuracy of complex piezoelectric nonlinear characteristics. Experiments have verified that the SLPI model not only has a higher modeling accuracy than the classic PI model, 
but also performs better in the compensation result. The use of the SLPI model reduced the error of maximum difference value on the outer loop by $10.0 \%$ and reduced the average error of the highest-level inner loop by $97.5 \%$. When compared to the classical PI inverse model, in spite of a small error increase on a vertex of the level-I loop, the error difference between single loops was reduced by $53.7 \%$; therefore, the overall average error was also reduced by $50.3 \%$. There was no error calculation in the two additional experiments; however, it was observed from the images that the SLPI model has a good modeling effect on all types of inverse-piezoelectric-effect-based nonlinear characteristics data. In the establishment of the SLPI model, no new operators were introduced; moreover, feedforward control was used, which was the compensation method in the previous principle. Through the analysis of the Play operator and PI model, the hysteresis advantage of the single-side Play operator was used, and the shortcomings of the PI model corresponding to multiple segments of one voltage were avoided. A single-loop independent hysteresis can be separated from the complex hysteresis characteristics. The segmented basis for modeling was to simplify the complex hysteresis nonlinearity to a single-loop model. When compared to the generalized PI model, this model corrected the hysteresis inclination deviation of the inner loop modeling and balanced the overall compensation result of the feedforward control. When compared to the previous segmented PI model, a reasonable separation basis was added to reduce the number of discontinuities. The SLPI model not only solved the phenomenon where a higher level of the inner ring leads to a lower precision of the modeling, but also enhanced the adaptability of the inherent characteristic curve of the outer ring material.

In this paper, it was still the inverse PI model that was used to solve the compensation control problem, which is a continuation of the previous work. The progress of the research in this paper provides a train of modeling thought and a theoretical basis for simplifying the complex characteristics. New concepts such as the endpoint and series of each hysteresis single loop proposed for nonlinear characteristic data provide sufficient reference value for subsequent research on real-time compensation. 
Acknowledgments The authors gratefully acknowledge the support of the National Natural Science Foundation of China through Grant No. 51975130, Key Research and Development Project of Liaoning Province through Grant No. 2017225016, and Natural Science Foundation of Liaoning Province through Grant No. 20180550002.

Compliance with ethical standards Not applicable

Conflicts of interest The authors declare that they have no conflict of interest.

\section{References}

1. Vasiljev, P., Mazeika, D., Kulvietis, G.: Modelling and analysis of omni-directional piezoelectric actuator. J. Sound Vib. 308, 867-878 (2007). doi:10.1016/j.jsv.2007.03.074 2. Park, G., Bement, M.T., Hartman, D.A., Smith, R.E., Farrar, C.R.: The use of active materials for machining processes: A review. Int. J. Mach. Tool. Manuf. 47, 2189-2206 (2007). doi:10.1016/j.ijmachtools.2007.06.002

3. Yuen, K.Y., Fleming, A.J., Moheimani, S.O.: Reza: A novel piezoelectric strain sensor for simultaneous damping and tracking control of a high-speed nanopositioner. IEEE Trans. Mechatron. 18, 1113 (2013). doi:10.1109/tmech.2012.2193895

4. Simu, U., Johansson, S.: Analysis of quasi-static and dynamic motion mechanisms for piezoelectric miniature robots. Sens. Actuators A 132, 632-642 (2006), https://doi.org/10.1016/j.sna.2006.02.023

5. Woody, S., Smith, S.: Design and performance of a dual drive system for tip-tilt angular control of a $300 \mathrm{~mm}$ diameter mirror. Mechatronics 16, 389-397 (2006). doi:10.1016/j.mechatronics.2006.03.006

6. Braunsmann, C., Schäffer, T.E.: High-speed atomic force microscopy for large scan sizes using small cantilevers. Nanotechnology 21, 225705 (2010). doi:10.1088/0957$4484 / 21 / 22 / 225705$

7. Ma, J., Tian, L., Li, Y., Yang, Z., Cui, Y., Chu, J.: Hysteresis compensation of piezoelectric deformable mirror based on Prandtl-Ishlinskii model. Opt. Commun. 416, 94-99 (2018). doi:10.1016/j.optcom.2018.02.001

8. Ge, P., Jouaneh, M.: Generalized Preisach model for hysteresis nonlinearity of piezoceramic actuators. Precis. Eng. 20, 99-111 (1997). doi:10.1016/S0141-6359(97)00014-7

9. Al Janaideh, M., Rakotondrabe, M., Al-Darabsah, I., Aljanaideh, O.: Internal modelbased feedback control design for inversion-free feedforward rate-dependent hysteresis compensation of piezoelectric cantilever actuator. Control Eng. Pract. 72, 29-41 (2018). doi:10.1016/j.conengprac.2017.11.001

10. Chen, W., Chen, S., Hu, Z., Tang, J., Li, H.: A novel dynamic model for the spiral bevel gear drive with elastic ring squeeze film dampers. Nonlinear Dyn. 98, 1081-1105 (2019). doi:10.1007/s11071-019-05250-9

\section{Springer}


11. Park, J., Moon, W.: Hysteresis compensation of piezoelectric actuators: The modified Rayleigh model. Ultrasonics 50, 335-339 (2010). doi:10.1016/j.ultras.2009.10.012

12. Ming, M., Feng, Z., Ling, J., Xiao, X.: Hysteresis modeling and feedforward compensation of piezoelectric nanopositioning stage with a modified Bouc-Wen model. Micro Nano Lett. 13, 1170-1174 (2018). doi:10.1049/mnl.2017.0793

13. Chen, Y., Shen, X., Li, J., Chen, J.: Nonlinear hysteresis identification and compensation based on the discrete Preisach model of an aircraft morphing wing device manipulated by an SMA actuator. Chin. J. Aeronaut. 32, 1040-1050 (2019). doi:10.1016/j.cja.2018.09.006

14. Stefanski, F., Minorowicz, B., Persson, J., Plummer, A., Bowen, C.: Non-linear control of a hydraulic piezo-valve using a generalised Prandtl-Ishlinskii hysteresis model. Mech. Syst. Signal Process. 82, 412-431 (2017). doi:10.1016/j.ymssp.2016.05.032

15. Ko, Y., Hwang, Y., Chae, M., Kim, T.: Direct identification of generalized PrandtlIshlinskii model inversion for asymmetric hysteresis compensation. ISA Trans. 70, 209-218 (2017). doi:10.1016/j.isatra.2017.07.004

16. Kuhnen, K.: Modeling, identification and compensation of complex hysteretic nonlinearities: A modified Prandtl-Ishlinskii approach. Eur. J. Control 9, 407-418 (2003). doi:10.3166/ejc.9.407-418

17. Cao, K., Li, R., Du, H., Ma, J.: Modeling and compensation of symmetric hysteresis in piezoceramic actuators. Results Phys. 13 (2019). doi:10.1016/j.rinp.2019.02.031, http://www.ncbi.nlm.nih.gov/pubmed/102095

18. An, D., Li, H., Xu, Y., Zhang, L.: Compensation of hysteresis on piezoelectric actuators based on tripartite PI model. Micromachines 9, 44 (2018). doi:10.3390/mi9020044

19. Yang, M., Gu, G., Zhu, L.: Parameter identification of the generalized Prandtl-Ishlinskii model for piezoelectric actuators using modified particle swarm optimization. Sens. Actuators A 189, 254-265 (2013). doi:10.1016/j.sna.2012.10.029

20. Gu, G., Yang, M., Zhu, L.: Real-time inverse hysteresis compensation of piezoelectric actuators with a modified Prandtl-Ishlinskii model. Rev. Sci. Instrum. 83 (2012).

doi:10.1063/1.4728575, http://www.ncbi.nlm.nih.gov/pubmed/065106

21. Yong, Y.K., Lu, T.F., Handley, D.C.: Review of circular flexure hinge design equations and derivation of empirical formulations. Precis. Eng. 32, 63-70 (2008).

doi:10.1016/j.precisioneng.2007.05.002

22. Gosavi, S.V., Kelkar, A.G.: Passivity-based robust control of Piezo-actuated flexible beam. In: Proceedings of the 2001 American control conference, 25-27 June pp. 2492-2497. doi:10.1109/acc.2001.946127. (Cat. No. 01CH37148), Arlington, VA, USA (2001)

23. Chu, C.L., Fan, S.H.: A novel long-travel piezoelectric-driven linear nanopositioning stage. Precis. Eng. 30, 85-95 (2006). doi:10.1016/j.precisioneng.2005.05.002 
24. Petroni, S., Tegola, C.L., Caretto, G., Campa, A., Passaseo, A., de Vittorio, M.D., Cingolani, R.: Aluminum nitride Piezo-MEMS on polyimide flexible substrates. Microelectron. Eng. 88, 2372-2375 (2011). doi:10.1016/j.mee.2011.02.080

25. Brokate, M., Sprekels, J.: Hysteresis and phase transitions. Springer, Berlin, Germany (1996)

26. Mokaberi, B., Requicha, A.A.G.: Compensation of scanner creep and hysteresis for AFM nanomanipulation. IEEE Trans. Automat. Sci. Eng. 5, 197-206 (2008).

doi:10.1109/TASE.2007.895008

27. Habineza, D., Zouari, M., le Gorrec, Y., Rakotondrabe, M.: Multivariable compensation of hysteresis, creep, badly damped vibration, and cross couplings in multiaxes piezoelectric actuators. IEEE Trans. Automat. Sci. Eng. 15, 1639-1653 (2018).

doi:10.1109/TASE.2017.2772221

28. Sun, Z., Song, B., Xi, N., Yang, R., Chen, L., Cheng, Y., Bi, S., Li, C., Hao, L.:

Systematic Hysteresis compensator design based on Extended Unparallel Prandtl-Ishlinskii model for SPM imaging rectification. IFAC-Papers Online 50, 10901-10906 (2017)

29. Liu, S., Su, C.Y.: A note on the properties of a generalized Prandtl-Ishlinskii model.

Smart Mater. Struct. 20 (2011). doi:10.1088/0964-1726/20/8/087003, http://www.ncbi.nlm.nih.gov/pubmed/087003(6)

30. Chi, Z.Q., Xu, Q.S.: Recent advances in the control of piezoelectric actuators. Int. J. Adv. Robot. Syst. 11, 182 (2014). doi:10.5772/59099

31. Leang, K.K., Zou, Q., Devasia, S.: Feedforward control of piezoactuators in atomic force microscope systems. IEEE Control Syst. 29, February, 70-82. doi:10.1109/MCS.2008.930922

32. Qin, Y., Tian, Y., Zhang, D., Shirinzadeh, B., Fatikow, S.: A novel direct inverse modeling approach for hysteresis compensation of piezoelectric actuator in feedforward applications. IEEE/ASME Trans. Mechatron. 18, June, 981-989.

doi:10.1109/TMECH.2012.2194301

33. Chi, Z., Xu, Q.: Precision control of piezoelectric actuator using fuzzy feedback control with inverse hysteresis compensation. In: Proceedings of the 10th IEEE international conference on Nano/Micro Engineered and Molecular Systems, Xi’an, China, 7-11 April pp. 219-224. doi:10.1109/nems.2015.7147414 (2015) 
Figures

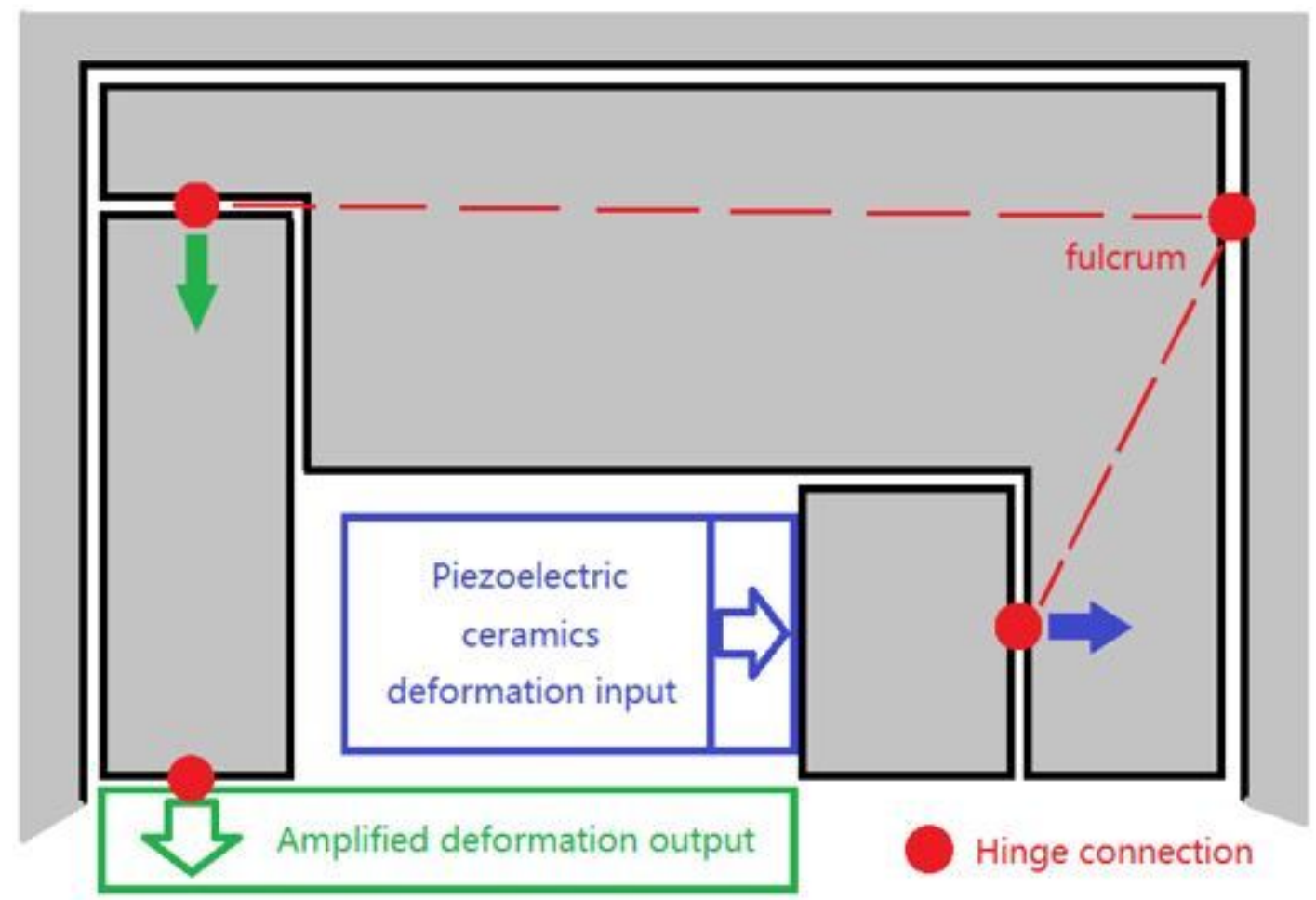

Figure 1

Schematic diagram of flexible hinge 



two degrees of freedom

1 electrical line holes

2 working device

3 piezoceramic stacks

4 flexible mechanism

5 location holes

Figure 2

Three-dimensional diagram of precision machining positioning device 




Figure 3

Experimental system for measuring the complex characteristics

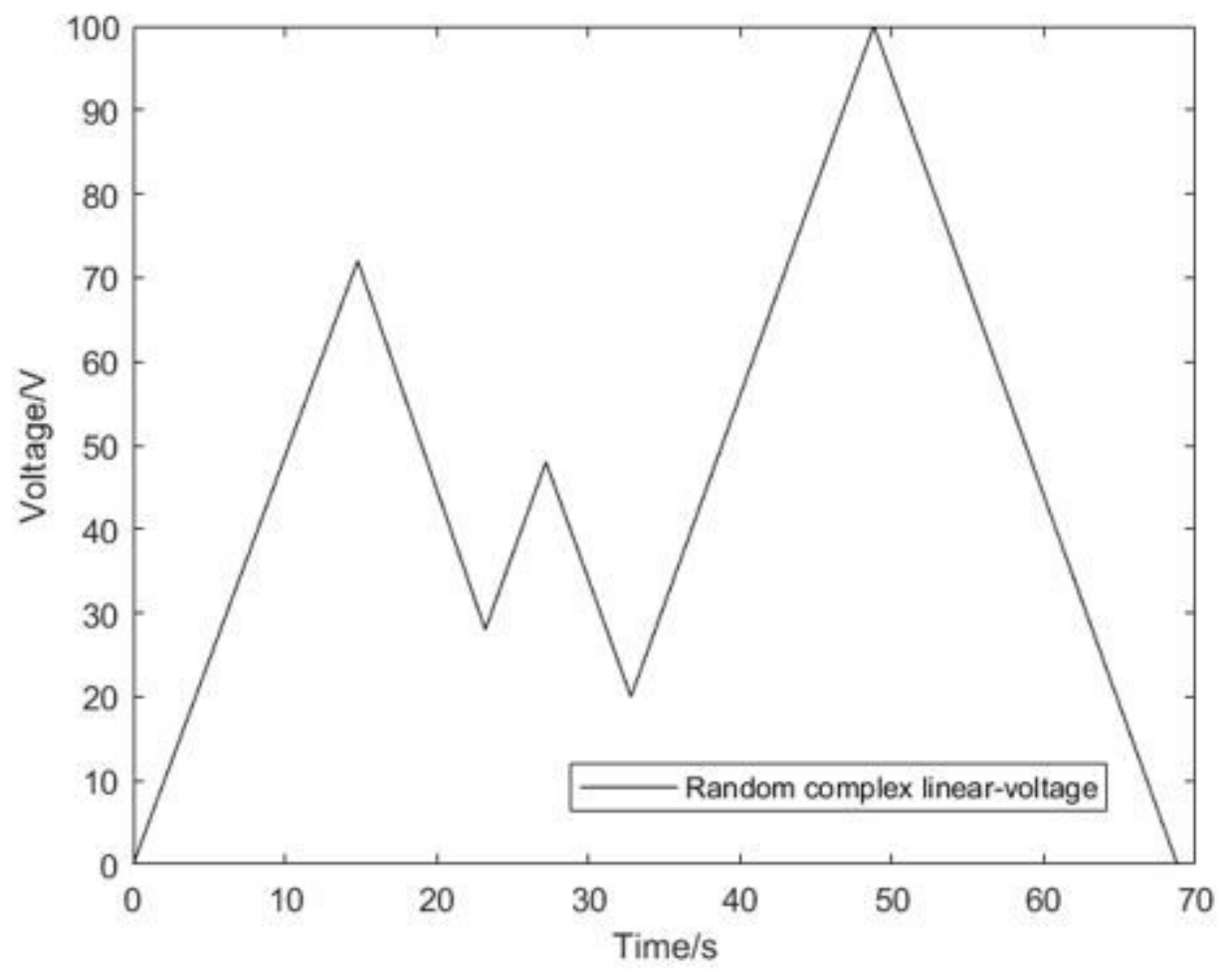

Figure 4 
Random complex voltage

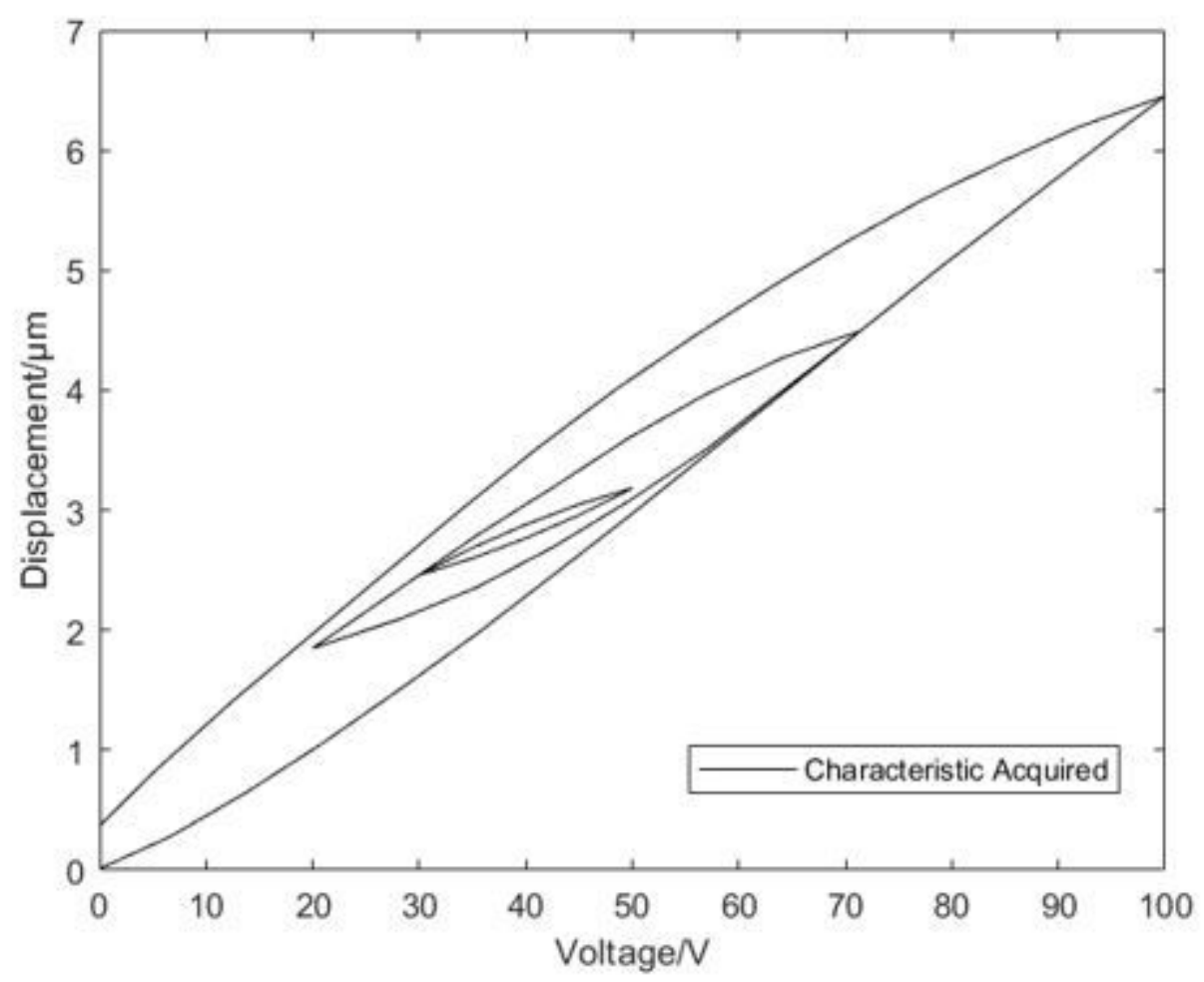

Figure 5

Nonlinear characteristics of complex voltage-displacement 



$\mathbf{c}$

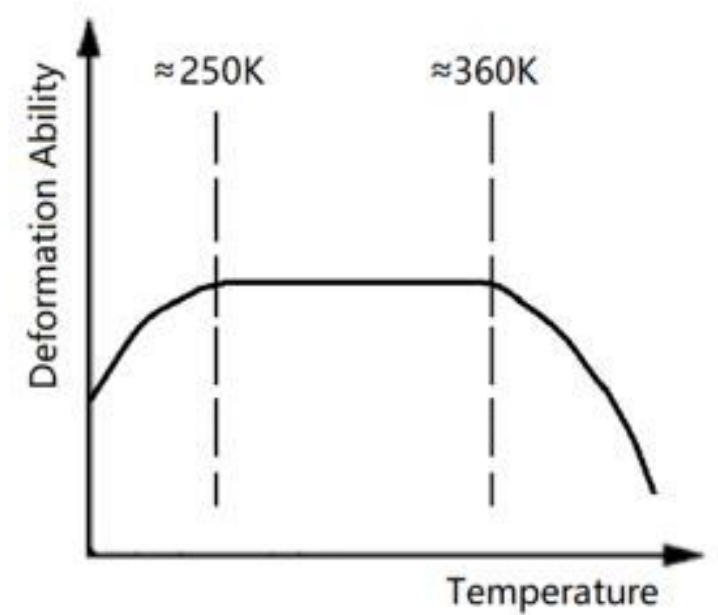

Figure 6

Schematic diagram of the causes of nonlinear characteristics: a Hysteresis characteristics; $b$ creep characteristics; c temperature characteristics 


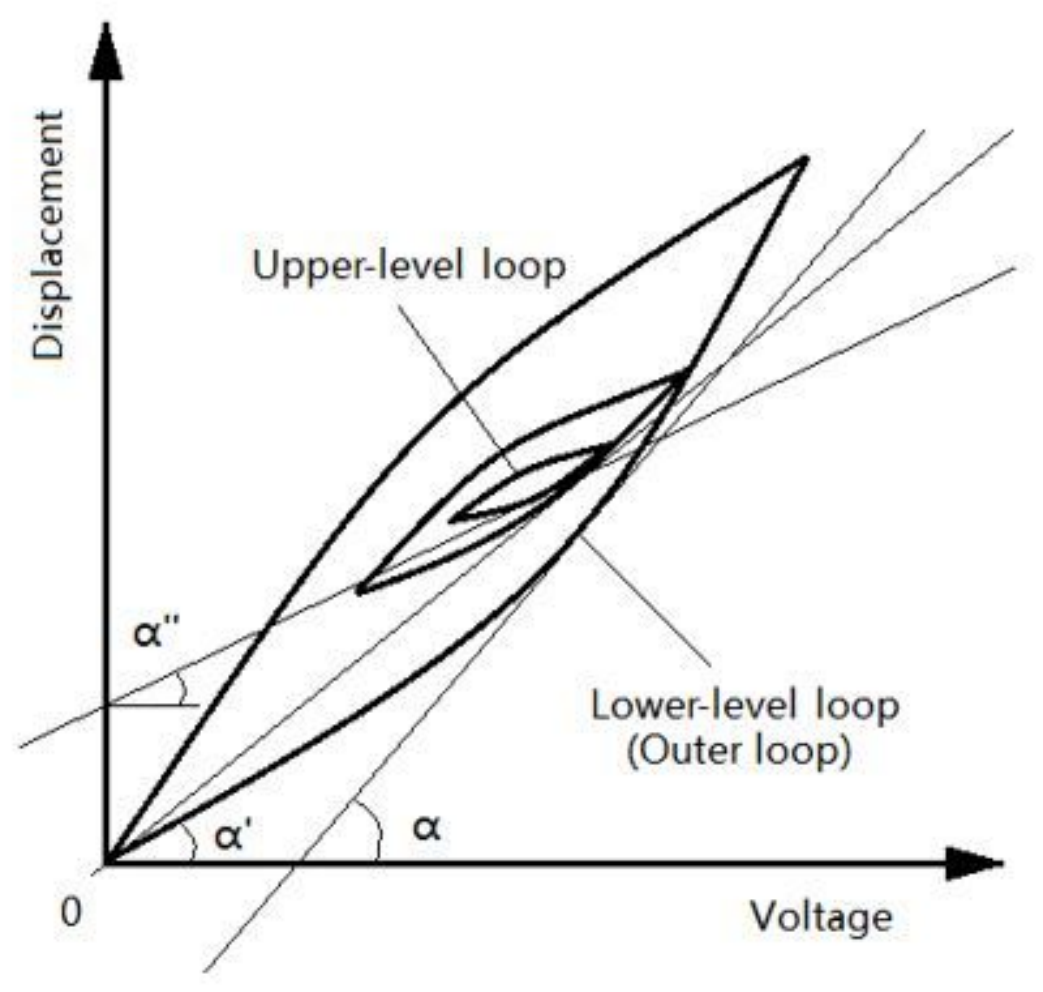

Figure 7

Differences in the hysteresis inclination angle of three hysteresis loops

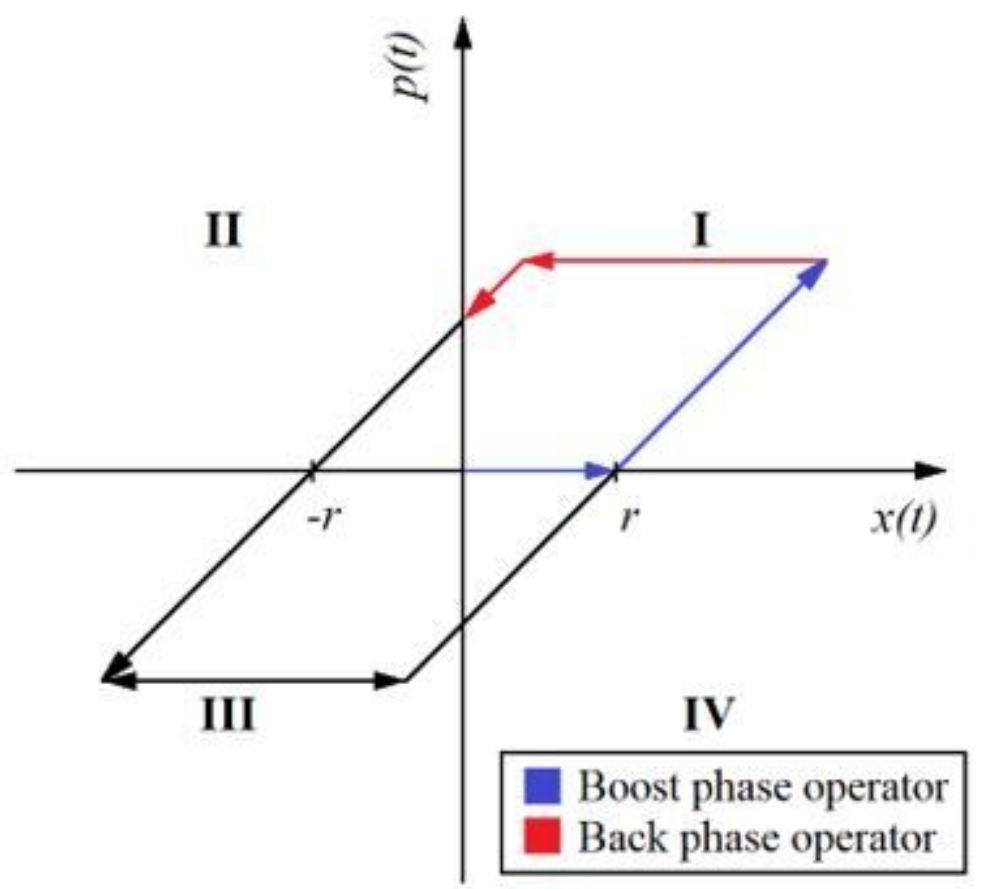

Figure 8

Play operator 
a

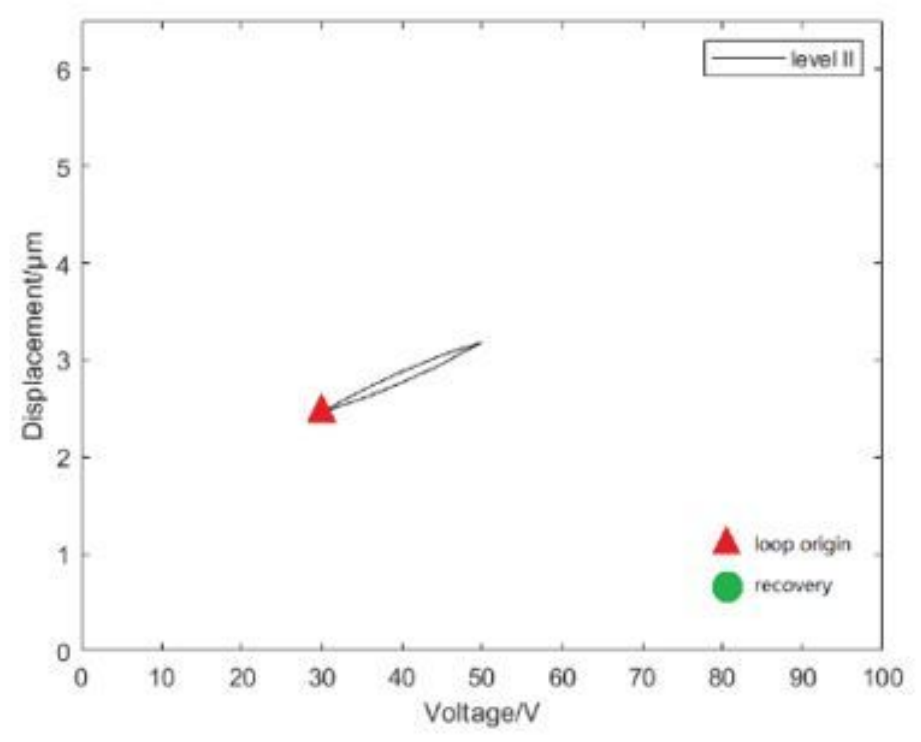

b

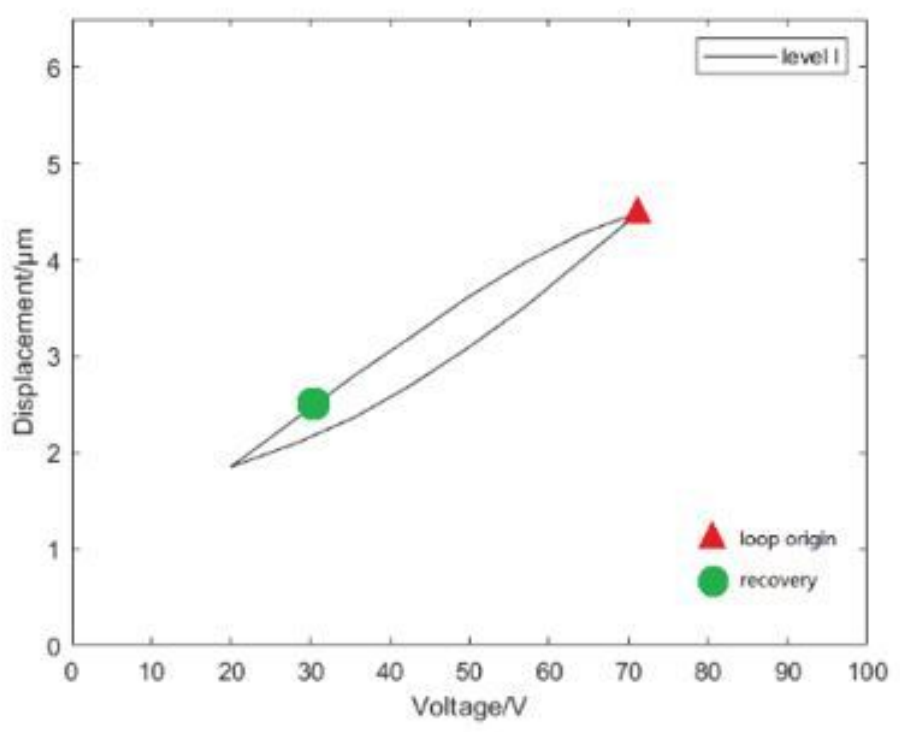

c



Figure 9

Single-loop-separation results: a Level-II single loop; b level-I single loop; c level-0 single loop 
a



b



c

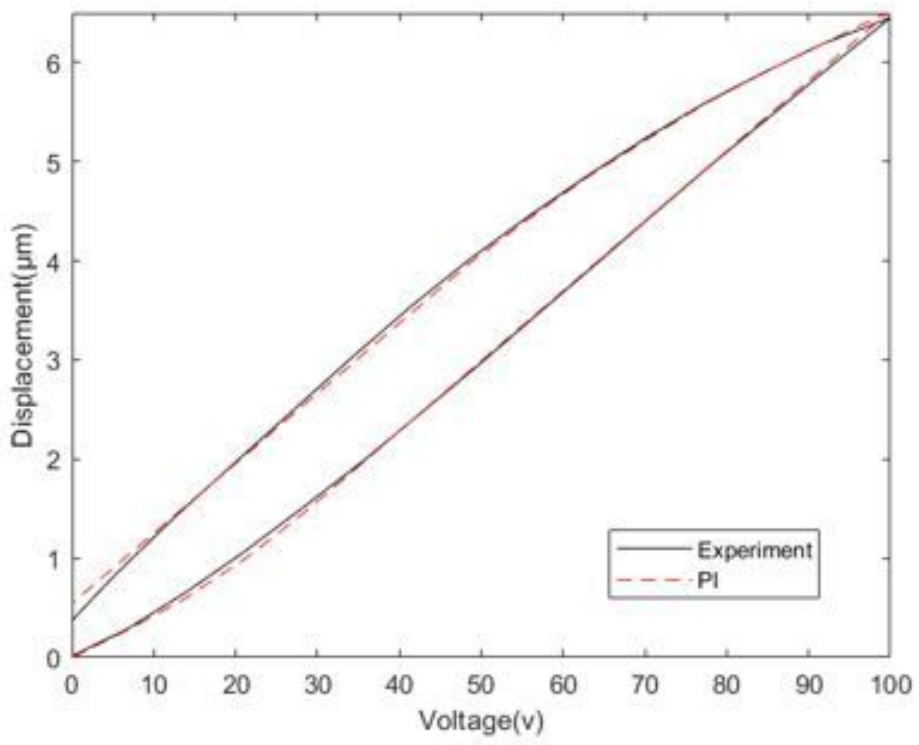

Figure 10

Single-loop Prandtl-Ishlinskii (PI) modeling: a Level-II single-loop modeling; b Level-I single-loop modeling; c Level-0 single-loop modeling 




Figure 11

Schematic diagram of feedforward compensation control 

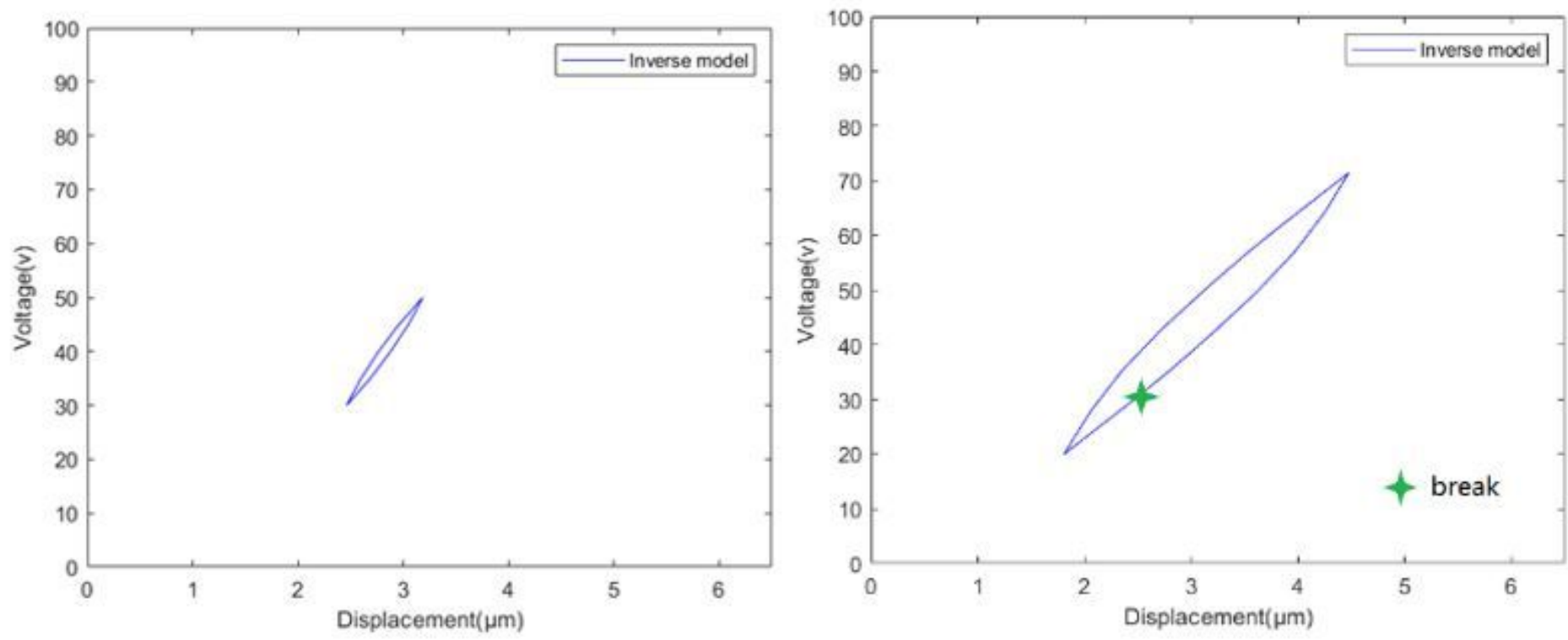

c

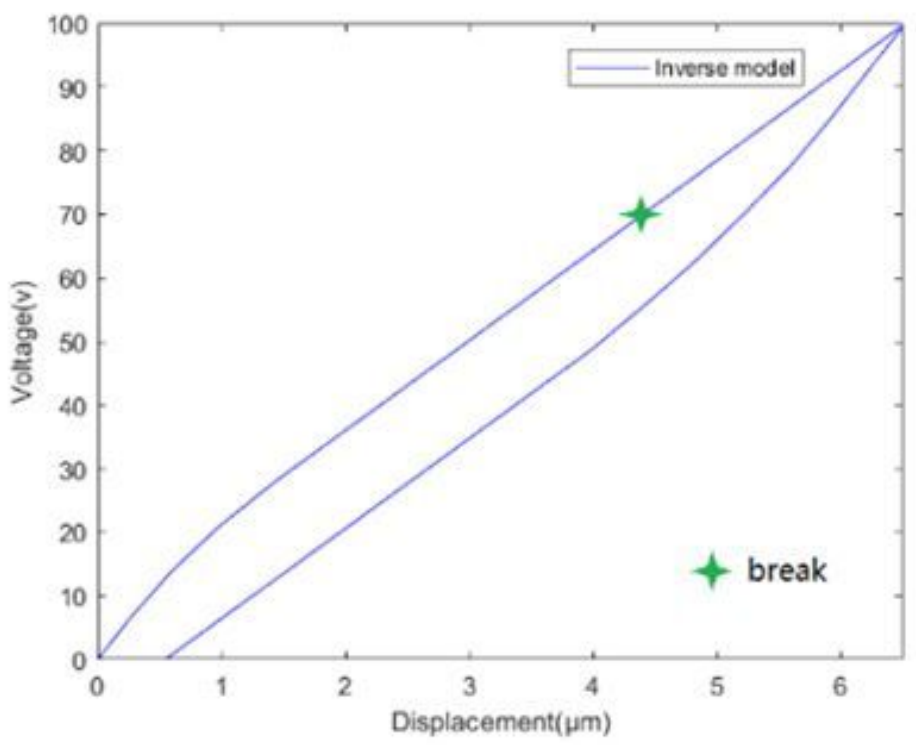

Figure 12

Single-loop inverse model: a Level-II single-loop inverse model; b level-I single-loop inverse model; c level-0 single-loop inverse model 


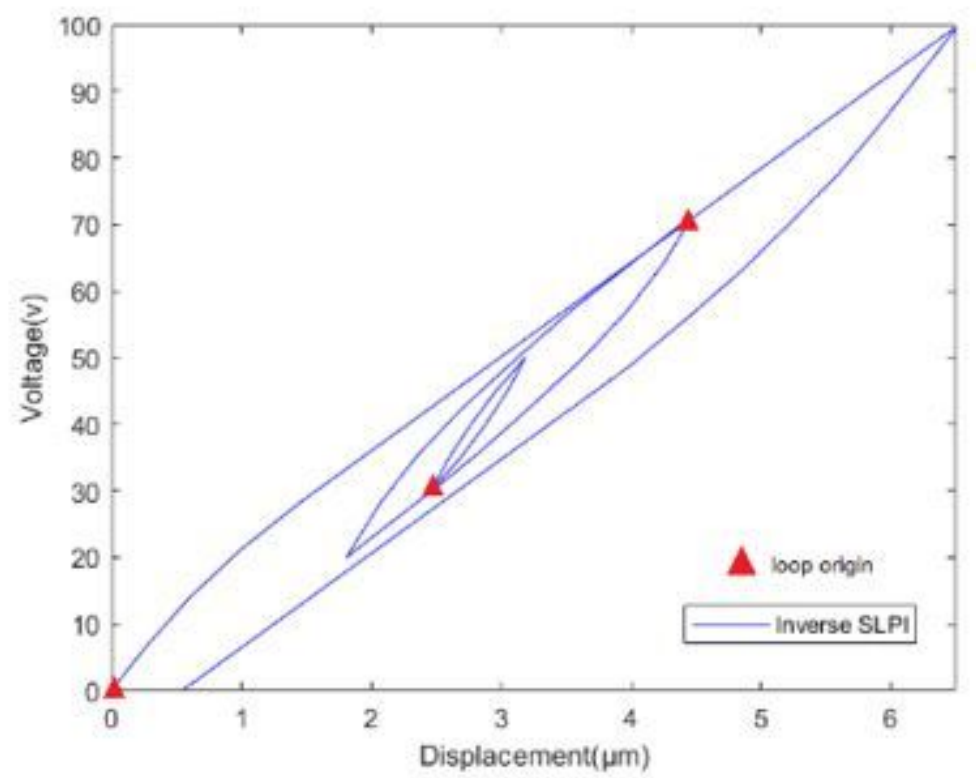

Figure 13

Separated Level-loop PI (SLPI) inverse model

a

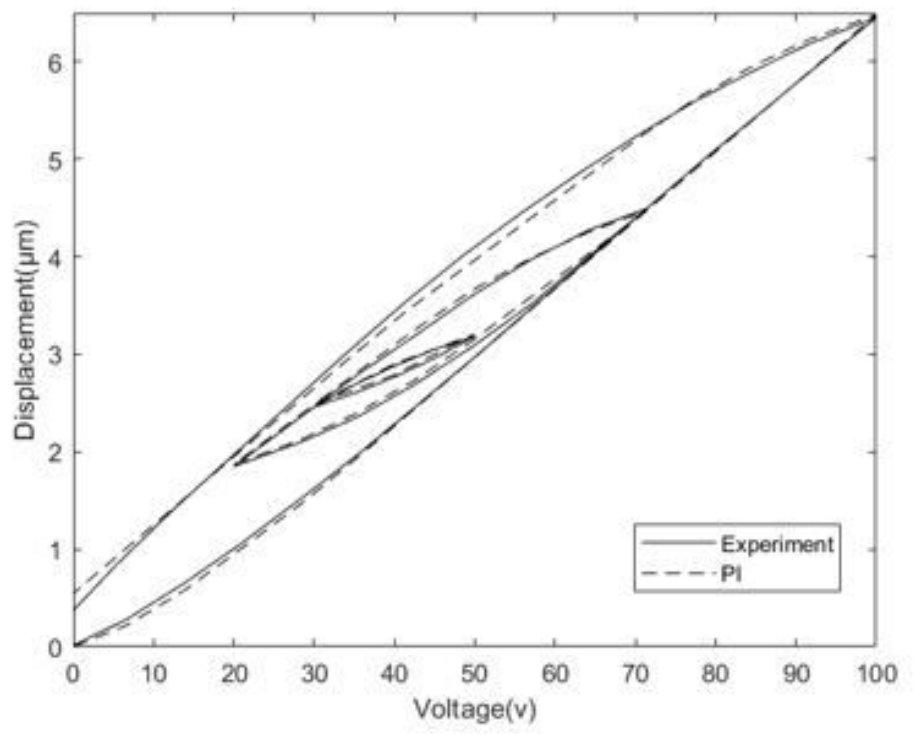

b



\section{Figure 14}

Modeling comparison diagrams: a Classical PI model comparison diagram; b gathered SLPI model comparison diagram 
a



c



b

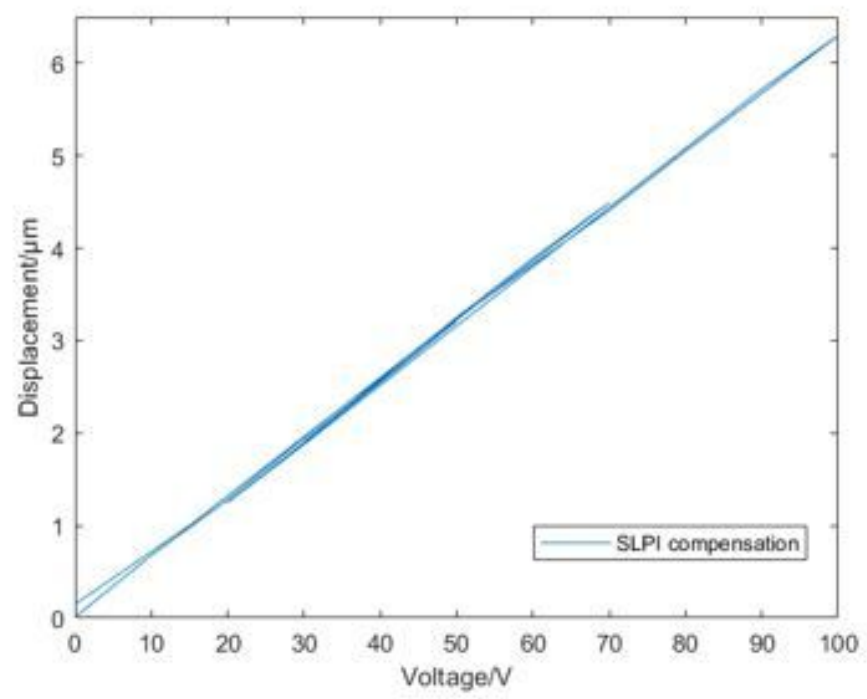

d



\section{Figure 15}

Compensation result diagrams: a Classical PI compensation, b SLPI compensation, c partially enlarged Fig. 15a, d partially enlarged Fig. 15b 


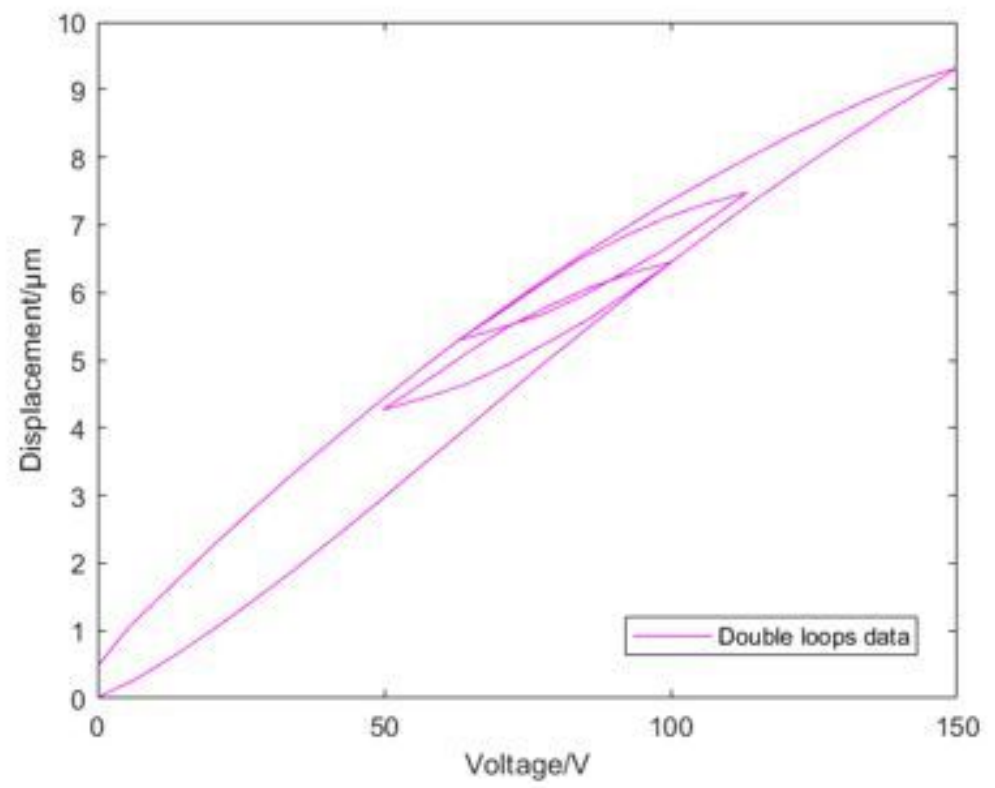

Figure 16

Double inner-loop hysteresis data

a

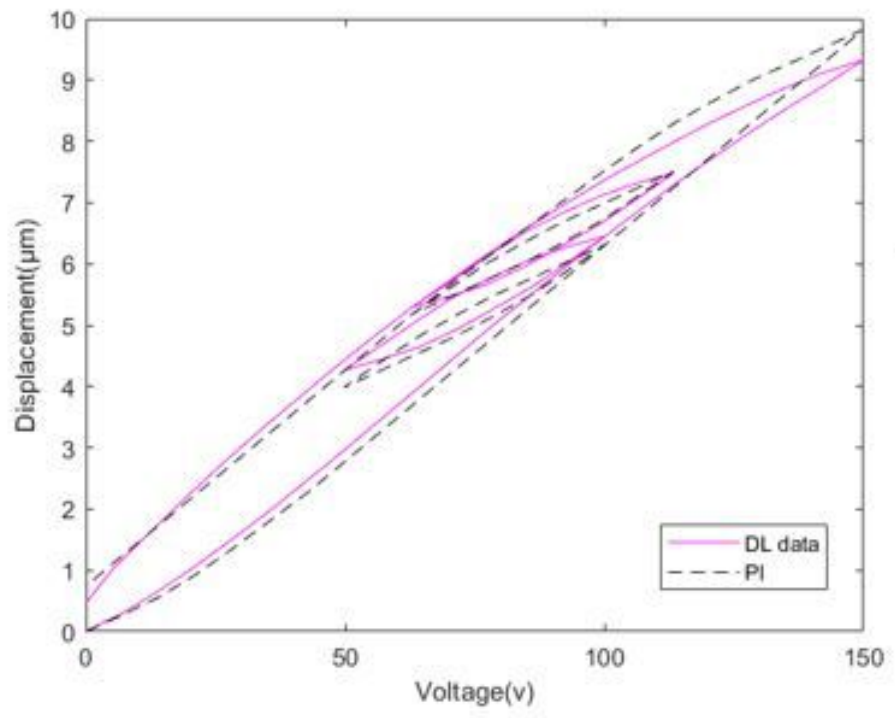

b

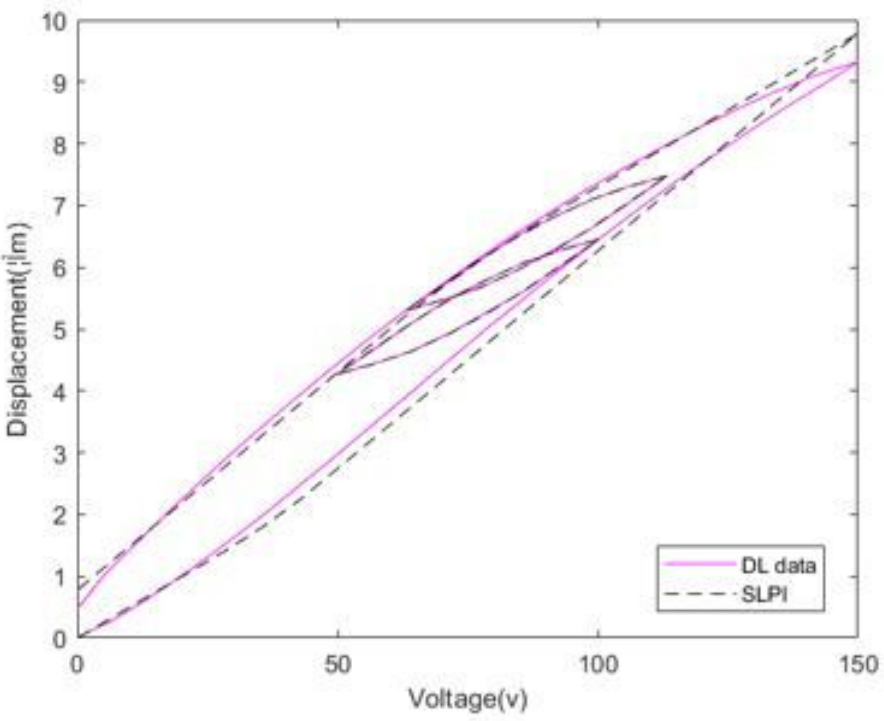

Figure 17

Comparison of double inner-loop hysteresis modeling: a Classical PI modeling; b SLPI modeling 


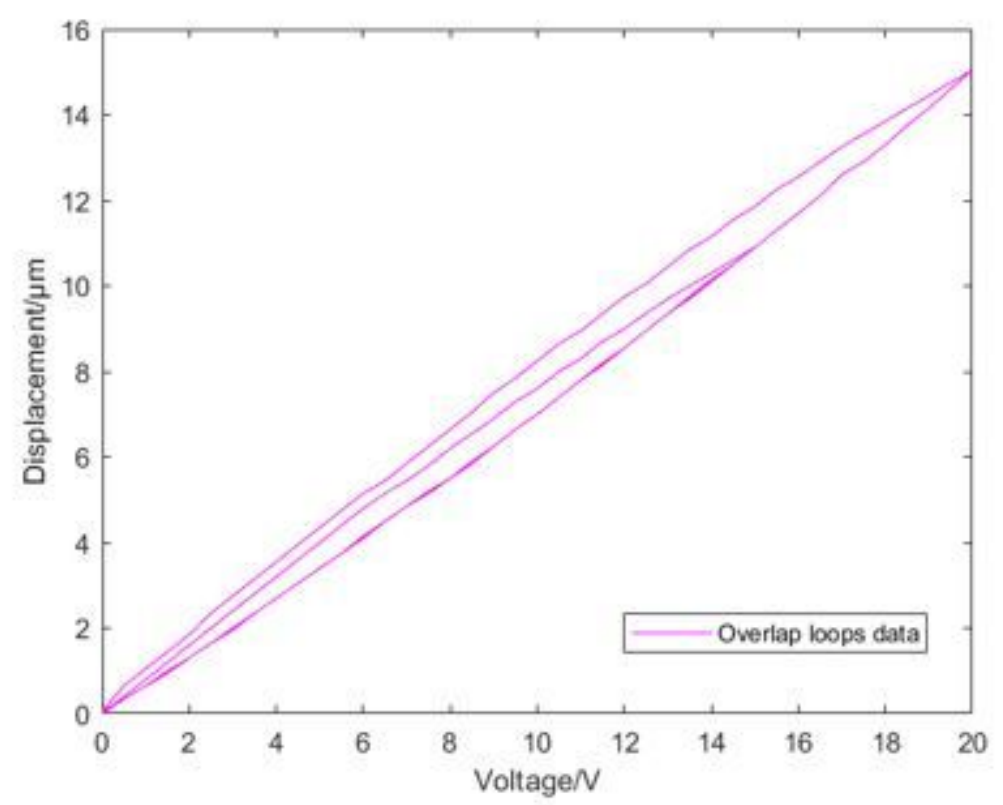

Figure 18

Overlapped hysteresis data

$\mathbf{a}$

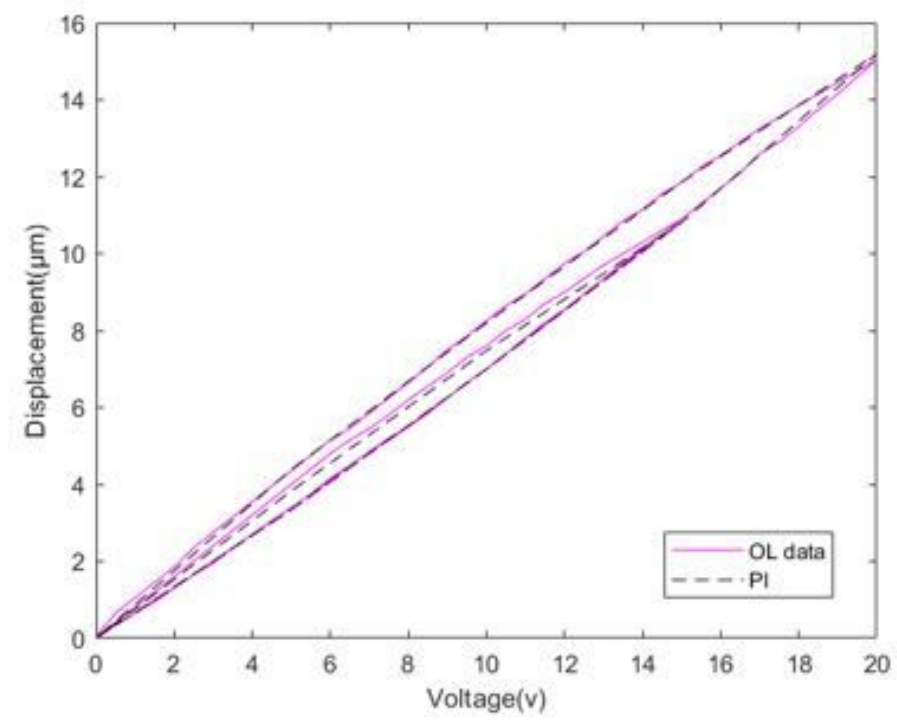

b

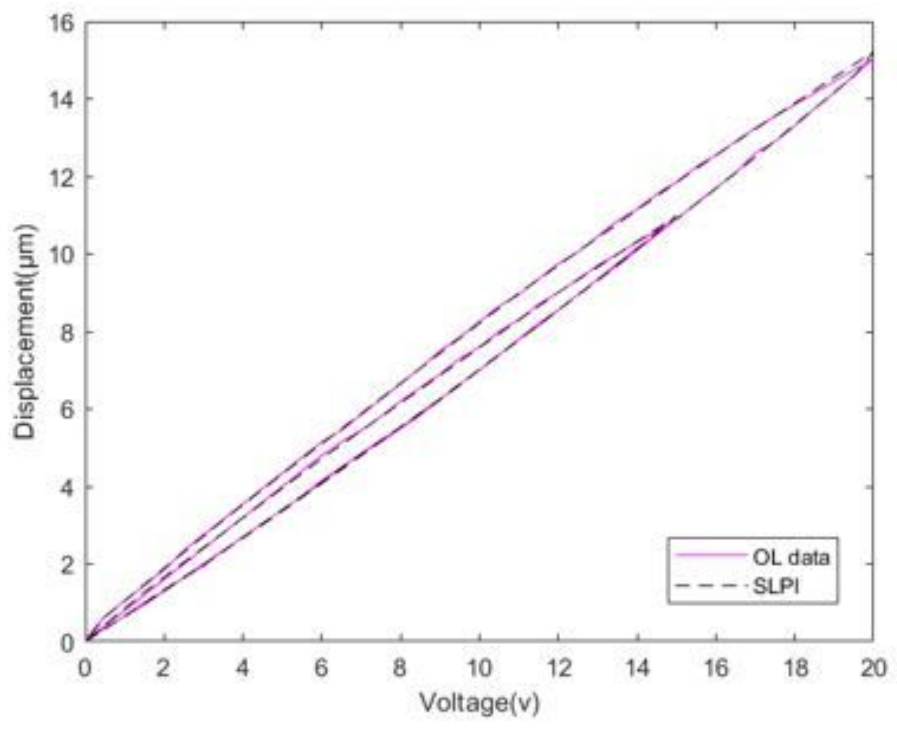

Figure 19

Comparison of overlapped hysteresis modeling: a Classical PI modeling; b SLPI modeling 